\title{
The dynamic epigenetic regulation of the inactive $X$ chromosome in healthy human $B$ cells is dysregulated in lupus patients
}

Sarah Pyfrom ${ }^{1 * *}$, Bam Paneru ${ }^{1 * *}$, James J. Knoxx ${ }^{2}$, Michael P. Cancro ${ }^{2}$, Sylvia Posso ${ }^{3}$, Jane H. Buckner ${ }^{3}$, and Montserrat C. Anguera ${ }^{1 *}$

${ }^{1}$ Department of Biomedical Sciences, School of Veterinary Medicine, University of Pennsylvania, Philadelphia, PA, USA

${ }^{2}$ Department of Pathology, Perlman School of Medicine, University of Pennsylvania, Philadelphia, PA, USA

${ }^{3}$ Benaroya Research Institute at Virginia Mason, Seattle, WA, USA

${ }^{* *}$ authors contributed equally

Running Title: Altered X-chromosome Inactivation in B cells of SLE patients

\author{
*Correspondence: \\ Montserrat C. Anguera \\ anguera@vet.upenn.edu \\ ${ }^{1}$ Department of Biomedical Sciences, School of Veterinary Medicine \\ University of Pennsylvania, Room 390EB \\ 3800 Spruce Street \\ Philadelphia, PA 19104 \\ Phone: (215) 898-0567 \\ ${ }^{2}$ Department of Pathology \& Laboratory Medicine, Perlman School of Medicine \\ University of Pennsylvania, Philadelphia, PA 19104 \\ ${ }^{3}$ Benaroya Institute at Virginia Mason \\ 1201 Ninth Avenue \\ Seattle, WA 98101
}

Keywords: XIST RNA, X-Chromosome Inactivation, SLE, human B cells, femalebiased autoimmunity, $\mathrm{X}$-linked gene expression, $\mathrm{XCl}$ escape, $\mathrm{H} 3 \mathrm{~K} 27 \mathrm{me}$, macroH2A, H2A-K119Ub. 


\section{$1 \quad$ ABSTRACT}

3 Systemic lupus erythematous (SLE) is a female-predominant disease characterized by

4 autoimmune B cells and pathogenic autoantibody production. Individuals with two or

5 more $X$ chromosomes are at increased risk for SLE, suggesting that $X$-linked genes

6 contribute to the observed sex-bias of this disease. To normalize X-linked gene

7 expression between sexes, one $X$ in female cells is randomly selected for transcriptional

8 silencing through $\mathrm{X}$-Chromosome Inactivation $(\mathrm{XCI})$, resulting in allele-specific

9 enrichment of epigenetic modifications, including histone methylation and the long

10 noncoding RNA XIST/Xist on the inactive $\mathrm{X}(\mathrm{Xi})$. As we have previously shown that

11 epigenetic regulation of the $\mathrm{Xi}$ in female lymphocytes from mice is unexpectedly

12 dynamic, we used RNA FISH and immunofluorescence to profile epigenetic features of

13 the $\mathrm{Xi}$ at the single cell level in human $\mathrm{B}$ cell subsets from pediatric and adult SLE

14 patients and healthy controls. Our data reveal that abnormal $\mathrm{XCl}$ maintenance in $\mathrm{B}$ cells

15 is a feature of SLE. Using single-cell and bulk cell RNA sequencing datasets, we found

16 that novel $\mathrm{X}$-linked immunity genes escape $\mathrm{XCl}$ in specific healthy human $\mathrm{B}$ cell

17 subsets, and that human SLE B cells exhibit aberrant expression of X-linked genes and

18 XIST RNA Interactome genes. Our data reveal that mislocalized XIST RNA, coupled

19 with a dramatic reduction in heterochromatic modifications at the Xi in SLE, predispose

20 for aberrant $\mathrm{X}$-linked gene expression from the $\mathrm{Xi}$, thus defining a novel genetic and

21 epigenetic pathway that affects X-linked gene expression in human SLE B cells and

22 likely contributes to the female-bias in SLE. 


\section{INTRODUCTION}

25 Systemic lupus erythematosus (SLE) is an incurable autoimmune disease with

26 multiorgan system manifestations. B cells contribute to various aspects of SLE by

27 secreting pathogenic autoantibodies, presenting autoantigens to T cells, and producing

28 inflammatory cytokines. In addition, the representation of B cell subsets changes in

29 SLE, which can accelerate the production of autoantibodies. In particular, CD27-

30 memory B cells ${ }^{1}, C D 19^{\text {hi }}$ XXCR3 $^{\text {hi }} B$ cells ${ }^{2}, C D 24^{-}$-activated naïve B cells ${ }^{3}$, and age-

31 associated $\mathrm{CD} 11 \mathrm{c}^{+} \mathrm{B}$ cells that express $\mathrm{T}-$ bet $^{4,5}$ are increased in autoimmunity. Thus, a

32 further understanding of the mechanisms involved in autoimmune B cell dysregulation is

33 critical for future efforts to control the development and progression of SLE.

35 Like many autoimmune diseases, SLE exhibits a strong female bias, with $85 \%$ of

36 patients being women. The underlying mechanisms responsible for this sex difference

37 are not well understood, yet it is clear that the genetics of the $\mathrm{X}$ chromosome impacts

38 disease susceptibility ${ }^{6}$. Indeed, individuals with two or more $\mathrm{X}$ chromosomes are at

39 increased risk for $\mathrm{SLE}^{7}$, suggesting that X-linked genes have a significant role in

40 disease. Immunity-related genes are enriched on the $X$ chromosome $e^{8,9}$, and some of

41 these genes are routinely overexpressed in SLE patient B cells ${ }^{10-13}$. In addition, mouse

42 models with $\mathrm{X}$-linked gene duplication (such as the BXSB-Yaa mouse model ${ }^{14,15}$ ) or

43 transgenic overexpression of either of the $\mathrm{X}$-linked genes $T / r 7^{16,17}$ or $B t k^{18,19}$ exhibit

44 disease resembling human SLE, with production of dsDNA autoantibodies. Thus, 
45 abnormal dosage or expression of particular X-linked genes is associated with SLE

46 disease in mice and humans.

48 Female mammalian cells with two $X$ chromosomes regulate $X$-linked gene expression

49 using $\mathrm{X}$-chromosome Inactivation $(\mathrm{XCl})$, in which one $\mathrm{X}$ is randomly selected for

50 transcriptional silencing to equalize gene expression between the sexes ${ }^{20,21}$. Numerous

51 epigenetic modifications, including histone methylation ${ }^{22,23}$, DNA methylation ${ }^{24,25}$, and

52 the long noncoding RNA XIST/Xist ${ }^{26-28}$ are enriched allele-specifically on the inactive $X$

$53(\mathrm{Xi})$, and maintain transcriptional repression of most of the X-chromosome. However,

54 some $\mathrm{X}$-linked genes escape $\mathrm{XCl}$, and human cells exhibit higher levels of $\mathrm{XCl}$ escape

$55(15-25 \% \text { of the } X \text { chromosome) compared to mice (3\% escape })^{29,30}$. While most

56 somatic cells maintain $\mathrm{XCl}$ with static enrichment of Xist RNA and heterochromatin

57 marks on the $\mathrm{Xi}$, we found that lymphocytes exhibit a unique dynamic localization of

58 these modifications to the Xi following stimulation ${ }^{31-33}$. These observations are likely to

59 be significant to pathogenesis, as we recently showed that $\mathrm{T}$ cells from SLE patients

60 have dispersed XIST RNA transcripts and aberrant overexpression of many X-linked

61 gene transcripts compared to T cells from healthy controls ${ }^{32}$.

62

63 In this study, we determined the epigenetic profile of the $\mathrm{Xi}$ in human B cell subsets at

64 the single-cell level, and found that typical heterochromatic modifications are missing

65 from the $\mathrm{Xi}$, suggestive of high levels of $\mathrm{XCl}$ escape across $\mathrm{B}$ cells. Remarkably, we

66 found mislocalized XIST RNA and reductions with the heterochromatin mark

67 H2AK119Ub at the Xi in activated B cells from pediatric and adult SLE patients, and 
68 accordingly, discovered aberrant gene expression profiles of X-linked genes in activated

69 SLE B cells. Our study demonstrates that the unique chromatin features of the $\mathrm{Xi}$ in

70 human $\mathrm{B}$ cells facilitates $\mathrm{XCl}$ escape, and we propose that impaired $\mathrm{XCl}$ maintenance in

71 SLE results in aberrant gene expression of $X$-linked genes, that may further contribute

72 to autoimmunity.

73

74

75 
RESULTS

Circulating human B cell subsets lack robust XIST RNA localization at the Xi

We previously reported that naïve B cells from female humans lack detectible XIST

81 RNA signals at the $\mathrm{Xi}$, and that naïve $\mathrm{B}$ cells from female mice are missing both Xist

82 RNA and enrichment of the heterochromatin modification H3K27me3 at the Xi. XIST

83 RNA localization patterns in lymphocytes can be classified into 4 groups ${ }^{31,32}$ : Type I

84 cells have robust XIST RNA localized on the Xi; Type II cells have diffuse XIST RNA

85 signals within a nuclear territory encompassing the $\mathrm{X}$ chromosome; Type III cells have

86 XIST RNA pinpoints across the nucleus; and Type IV cells lack XIST RNA signals (Fig.

87 1A). To determine whether XIST RNA and the heterochromatin modification H2AK119-

88 ubiquitin (H2AK119Ub) were missing from the $\mathrm{Xi}$ in human B cell subsets, we isolated

89 circulating naïve B $\left(\mathrm{CD}_{19}{ }^{+} \mathrm{CD} 10^{-} \mathrm{CD} 21^{+} \mathrm{IgD} \mathrm{D}^{+}\right)$, memory $\mathrm{B}\left(\mathrm{CD} 19^{+} \mathrm{CD}^{+} 7^{+}\right)$, plasma

$90\left(\mathrm{CD}^{+} \mathrm{CD}^{+} \mathrm{C}^{+}\right)$, and age-associated B cells (ABCs; CD19 $\left.{ }^{+} \mathrm{CD} 11 \mathrm{c}^{+}\right)$from healthy

91 human donors for sequential XIST RNA FISH and immunofluorescence (IF).

92 Remarkably, we found that both XIST RNA transcripts and H2AK119Ub foci were

93 missing from the $X i$ in naïve $B$ cells, plasma cells, and ABCs (Fig. 1B). Memory B cells

94 had dispersed XIST RNA signals across the nucleus, yet also lacked H2AK119Ub foci

95 (Fig. 1A). We quantified the XIST RNA localization patterns for human B cell subsets

96 and found that naïve $B, A B C s$, and plasma B cells are predominantly Type IV, and

97 memory B cells were mostly Type III with some Type II patterns (one-way ANOVA for

98 Type II, Type III, Type IV p < 0.05; Fig. 1C). All four B cell subsets examined lacked 
99 detectable H2AK119Ub foci (Fig. 1D), including memory B cells that displayed Type III

100 XIST RNA pinpoints across the nucleus. As a previously published RNAseq dataset ${ }^{34}$

101 revealed that $X I S T$ is continuously expressed in naïve, memory, and ABC cells (Fig.

102 1E), our findings indicate that XIST RNA localization and transcription of the XIST gene

103 are genetically uncoupled in human B cell subsets.

XIST RNA and heterochromatin modifications H2AK119Ub and H3K27me3 are

localized at the $\mathrm{Xi}$ after in vitro activation of mature naïve human B cells

108 While naïve $B$ cells from female mice lack Xist RNA and heterochromatin mark

109 enrichment on the $\mathrm{Xi}$, we have shown that these epigenetic modifications return to the

$110 \mathrm{Xi}$ at 24-30 hrs post stimulation ${ }^{35}$. Here, we determined if the dynamic localization of

111 XIST RNA is similarly observed in healthy naïve and memory B cells stimulated in vitro

112 using CpG for 3 -7 days. Using RNA FISH, we find that XIST RNA transcripts were first

113 detected in naïve B cells at 1-2 days post-stimulation, and signals decreased by 3-4

114 days post-stimulation (Fig. 2A). The efficiency of in vitro stimulation was assessed by

115 CD86+ staining, with $\sim 50-60 \%$ of $B$ cells being positive for this marker in each XIST

116 RNA experiment (Fig. 2B). We quantified the XIST RNA localization patterns during

117 human B cell activation and found that day 2 stimulated B cells had the highest levels of

118 Type I and Type II XIST RNA localization patterns (Fig. 2C), with such patterns

119 appearing at day 1 post-stimulation. Type III XIST RNA localization patterns were

120 predominant at days 3-7 post in vitro activation (Fig. 2C). XIST RNA transcript levels

121 were relatively similar between naïve and in vitro stimulated B cells (Supplemental 
122 Figure 1), as previously observed in mouse B cells ${ }^{35}$ and reflecting uncoupled XIST

123 transcription and localization to the Xi. Similar analysis of circulating memory B cells

124 revealed that Type I and II XIST RNA patterns predominated after 3 days of culture

125 post-stimulation for memory B cells (Fig. 2D, 2E). In sum, in vitro activation using CpG

126 stimulates the return of XIST RNA transcripts to the $\mathrm{Xi}$ in both naïve and memory $\mathrm{B}$

127 cells.

129 We next asked whether XIST RNA recruitment to the Xi coincided with the enrichment 130 of heterochromatic foci typical of the Xi in somatic cells ${ }^{22}$. Circulating naïve B cells were 131 activated with CpG for 2 days, and then used for sequential XIST RNA FISH followed by 132 IF using antibodies for H3K27me3 and H2AK119Ub. We quantified the number of nuclei 133 that exhibited co-localization of XIST RNA with a heterochromatic focus (Fig. 3). The 134 majority of activated B cells (40-80\%) contained a focus that co-localized with Type I 135 XIST RNA patterns and either H2AK119Ub (Fig. 3A) or H3K27me3 (Fig. 3B). There 136 were very few nuclei with XIST Type IV patterns (purple bars), and a focus with either 137 H2AK119Ub or H3K27me3 (2-4\%), suggesting that XIST RNA localization to the Xi may 138 be necessary for enrichment of these repressive modifications. Return of epigenetic 139 marks to the Xi during human B cell activation occurs in one phase in which both XIST 140 RNA and heterochromatin modifications appear concurrently at the Xi beginning at day 1411 post-stimulation using CpG, with peak enrichment occurring at day 2.

143 Next, we asked whether the histone variant macroH2A, which is also typically enriched 144 on the $\mathrm{Xi}$ in fibroblasts, was localized to the $\mathrm{Xi}$ of in vitro activated healthy human $\mathrm{B}$ 
145 cells. We observed very few macroH2A1 foci in these cells, with approximately $10 \%$ co-

146 localization of XIST RNA with a focus of macroH2A1 (Supplemental Figure 2A, 2B).

147 Use of qRT-PCR revealed expression of both transcript variants, but these levels still

148 remained below those observed in human female fibroblasts (Supplemental Figure 2C).

149 We also investigated whether the active chromatin modification H3K4me3 was depleted

150 within the territory of the $\mathrm{Xi}$ in activated $\mathrm{B}$ cells, as typically observed in female

151 fibroblasts ${ }^{36,37}$. Using sequential XIST RNA FISH followed by IF, we observed the

152 characteristic H3K4me3 'holes', reflecting active transcription, which overlapped with

153 XIST RNA Type I and II signals in 70-85\% of the nuclei (Supplemental Fig. 3). In sum,

154 the chromatin of the $\mathrm{Xi}$ in $\mathrm{CpG}$ activated human B cells is enriched for some, but not all,

155 silent and active chromatin modifications, underscoring important differences with other 156 somatic cells.

158 Single-cell transcriptional profiling of human B cell subsets reveals cell-type specific biallelic expression of X-linked genes

161 The absence of XIST RNA and heterochromatic modifications H2AK119Ub, H3K27me3,

162 and macroH2A from the $\mathrm{Xi}$ in circulating $\mathrm{B}$ cell subsets suggests that there may be 163 either increased overall transcription from this chromosome or that the number of genes

164 that escape from $\mathrm{XCl}$ would be increased. To investigate this possibility, we queried 165 single-cell RNA sequencing (scRNAseq) data from a recent study ${ }^{38}$ examining 117 166 human B cells isolated from a healthy female donor. These cells were sorted by surface 167 markers and consist of 30 memory B cells, 30 naïve B cells, 30 plasmablasts, and 27 
168 transitional B cells. We determined the X-linked SNP expression for genes in each cell using a SNP detection threshold of at least 10 reads/SNP (Fig. 4A). Each X-linked gene containing a SNP was called 'monoallelic' if greater than $90 \%$ of the reads had the

171 same SNP, otherwise the gene was called 'biallelic'. We detected 6816 individual X-

172 linked SNPs, and 391 unique X-linked genes that were expressed across the B cell

173 subsets (Fig. 4A; Supplemental Tables 1, 2). We observed novel cell-type specific XCl

174 escape with higher levels of biallelic expression in memory B cells (98 biallelic genes)

175 and plasmablasts (122 biallelic genes) compared to transitional B and naïve B cells

176 (light blue, Fig. 4B; Supplemental Table 3). We detected a total of 190 X-linked genes

177 that escape $\mathrm{XCl}$ across all $\mathrm{B}$ cell subsets and $77 \%$ of these genes were novel $\mathrm{XCl}$

178 escape genes, as they had not been reported previously (Supplemental Table 3). We

179 also observed expression of 53 X-linked immunity-related genes across the B cell

180 subsets, and found that 38 of these genes (72\%) were biallelically expressed (light blue,

181 Fig. 4C; Supplemental Table 4). We summarized the expression status for the X-linked

182 immunity-related genes across the four human B cell subsets in Figure 4D. Biallelic

183 expression (light blue) of $D D X 3 X$ in all four B cell subsets was expected, as $D D X 3 X$

184 ubiquitously escapes $\mathrm{XCl}$ in multiple tissues ${ }^{30}$. While NONO, AP1S2, TSC22D3,

185 AP1S2, IL2RG, SASH3, MSN, and CYBB also escaped $\mathrm{XCl}$ across all $4 \mathrm{~B}$ cell subsets,

186 the significance of this biallelic expression is unknown as overexpression of these

187 genes has not been reported in autoimmune diseases. As expected, XIST was

188 exclusively monoallelic (dark blue) across all B cells (Fig.4D), given its selective

189 expression from the $\mathrm{Xi}$. We also detected variable $\mathrm{XCl}$ escape of TLR7 in naïve $\mathrm{B}$ cells

190 and plasmablasts, which has been observed previously by our group and others ${ }^{31,39}$. 
191 Interestingly, we also observed variable $\mathrm{XCl}$ escape for $B T K$, which is notable because

192 dosage imbalances of this gene are associated with lupus-like phenotypes ${ }^{18,19}$. In sum,

193 human B cells exhibit cell-type specific XCl escape of important immune regulatory

194 genes that could potentially contribute to sex-dependent differences in cellular function, 195 thereby impacting autoimmune disease.

197 Pediatric SLE patient B cells have missing or mislocalized XIST RNA transcripts 198 from the Xi and lack H2AK119Ub foci

200 Cell lines derived from SLE patient B cells exhibited differences in XIST RNA 201 localization patterns compared to cell lines from age-matched, healthy individuals ${ }^{31}$. 202 Here, we investigated whether primary naïve B cells from SLE patients would also have 203 mislocalized XIST RNA patterns. Naïve B cells isolated from pediatric SLE patients in 204 disease remission (SLE disease activity index [SLEDAI] score 0-1) were stimulated in 205 vitro using CpG for 2 days, and then used for XIST RNA FISH analyses. We quantified 206 the percentage of each type of XIST RNA localization pattern for both circulating naïve 207 B cells and activated B cells that had been stimulated in vitro (Supplemental Figure 4).

208 There were no significant differences between XIST RNA localization patterns for 209 circulating naïve B cells from pediatric SLE patients and age-matched healthy controls 210 (Supplemental Figure 4A). In contrast, we found that there were very few examples of 211 Type I and significantly reduced levels of Type II XIST RNA patterns for pediatric SLE in 212 vitro stimulated B cells compared to healthy controls (Fig. 5A, 5B, Supplemental Figure 213 4B; $\mathrm{p}<0.0001$, and $\mathrm{p}=0.0002)$. In vitro activated SLE patient $\mathrm{B}$ cells also had 
214 significantly higher levels of Type IV XIST RNA patterns, where nuclei lack detectable

215 XIST signals (Fig. 5B, Supplemental Figure 4B; $p=0.008$ ). Aberrant XIST RNA

216 localization patterns in activated pediatric SLE B cells were not a result of impaired or

217 ineffective stimulation using CpG (a TLR9 agonist), as SLE B cells had similar levels of

218 the activation marker CD86 (Supplemental Figure 4C). XIST RNA localization patterns

219 were also disrupted in SLE patient memory B cells compared to healthy controls, with

220 predominantly Type III and Type IV patterns (Supplemental Figure 4D; $p=0.004$ ). To

221 further assess if enrichment of the heterochromatic modification H2AK119Ub was also

222 affected, we performed IF for H2AK119Ub on circulating and in vitro activated B cells

223 from pediatric SLE patients and healthy controls. We observed a significant reduction in

$224 \mathrm{H} 2 \mathrm{~K} 119 \mathrm{Ub}$ foci in the activated B cells from pediatric SLE patient samples relative to

225 healthy controls (Fig. 5C; $p=8.9 E-6$ ). Circulating naïve B cells from both SLE patients

226 and healthy controls lacked detectible H2AK119Ub foci, as expected (Fig. 1). In sum,

227 mislocalization of XIST RNA and near-absence of H2AK119Ub foci on the Xi is a

228 feature of activated pediatric SLE patient B cells in disease remission.

B cells from adult SLE patients have aberrant XIST RNA localization patterns and

231 significant reductions in H2AK119Ub enrichment on $\mathrm{Xi}$, irrespective of disease

232 activity

233

234 We initially confirmed that that CD27- isolated cells from SLE patients had relatively

235 similar representation of all B cell populations relative to healthy controls (Supplemental

236 Figure S5). Similar to in vitro activated pediatric SLE patient B cells, in vitro activated B 
237 cells from adult SLE patients had significantly fewer Type I $(p<0.0001)$ and Type II $(p<$

238 0.0001) XIST RNA patterns, and significantly more Type IV patterns $(p<0.0001)$,

239 signifying abnormal XIST RNA localization patterns in B cells from adult SLE patients

240 (Figures 6A, 6B; Supplemental Figure 6). In contrast to the pediatric SLE population,

241 about half of the adult SLE patients had similar levels of Type I and Type IV XIST RNA

242 patterns as healthy controls (Fig. 6B). Co-localization of XIST RNA with H2AK119Ub

243 foci were significantly reduced in adult SLE patient B cells compared to healthy controls

244 (blue bars, $p<0.0001$; Fig. 6C), and cells containing an H2AK119Ub focus independent

245 of XIST RNA signals (Types I, II) were absent in SLE samples (orange bars). Activation

246 of naïve B cells following stimulation with CpG (as determined by CD86+ levels) was not

247 significantly affected in adult SLE samples (Supplemental Figure 6B). In sum,

248 enrichment of both XIST RNA and the heterochromatic modification H2AK119Ub at the

249 Xi are significantly reduced for in vitro activated adult SLE B cells.

250

251 We next asked whether the distribution of XIST RNA patterns correlated with SLE

252 disease activity (SLEDAl values), patient medications, age, or disease duration. For

253 these analyses, we removed patients with known comorbidities, including thyroid

254 illnesses (such as Grave's disease) as they exhibit greater Type III and fewer Type IV

255 XIST RNA patterns (Supplemental Figure S7; left panels). Analysis of each XIST RNA

256 localization pattern and the 6 medications typically used to treat SLE symptoms

257 demonstrated that only hydroxychloroquine showed significant correlation with Type I

258 XIST RNA localization patterns, comparable to healthy controls (Supplemental Fig.

259 7A,7E). We then performed multiple linear regressions between continuous patient 
260 metrics (age at sample draw, SLEDAI score, anti-nuclear antibody titer, and disease

261 duration) and the percentage of Type I-IV XIST RNA localization patterns

262 (Supplemental Figure S8). Of all supplied metrics, SLE patient age was the only one

263 that correlated positively with the Type IV XIST RNA patterns ( $p$-value: $0.030, \mathrm{R}^{2}: 0.214$;

264 Supplemental Fig. S8B). Healthy control samples did not exhibit a similar correlation

265 (Supplemental Fig. S8B). Taken together, activated B cells from adult SLE patients

266 exhibit aberrant XIST RNA localization and reduced H2AK119Ub enrichment on the Xi,

267 irrespective of disease activity but correlated with patient age, suggestive of

268 impairments with gene expression on this chromosome.

270 Activated B cells from SLE patients exhibit abnormal expression of X-linked

271 genes and XIST RNA Interactome genes

272

273 Because SLE activated B cells from both pediatric and adult SLE patients exhibited

274 abnormal XIST RNA localization and reduced/missing H2AK119Ub enrichment at the

$275 \mathrm{Xi}$, we asked whether these changes were also associated with abnormal X-linked gene

276 expression. To answer this, we utilized a previously published RNA sequencing dataset

277 (GSE118254) that profiled activated B cells in circulation (CD19+lgD+CD27-

278 MTG+CD24-CD38-) in seven female SLE patients and six healthy controls ${ }^{40}$. In this

279 dataset, we found that 53 X-linked genes were differentially expressed in activated B

280 cells from SLE patients, and 18 of these genes were overexpressed (Figure 7A). Among

281 the 53 differentially expressed X-linked genes, 4 have known immune functions (genes

282 in orange), and these genes exhibited $\mathrm{XCl}$ escape in at least $1 \mathrm{~B}$ cell subset (Fig. 4D, 
283 Supplemental Table S3). Comparison of the 53 X-linked genes altered in SLE with our

284 results from Supplemental Table 3 and published lists of $\mathrm{XCl}$ escape genes from

285 various cell types ${ }^{30}$ indicate that the majority of the genes in Fig. 7A (all the genes in

286 color) may escape $\mathrm{XCl}$ in activated B cells. Notably, 18 X-linked genes were

287 significantly upregulated in SLE patient activated B cell samples (red genes), and few of

288 the downregulated genes (blue genes) passed the significance threshold (Fig. 7B).

289 Unexpectedly, we found that the majority of these putative $\mathrm{XCl}$ escape genes are

290 downregulated in SLE B cells (Fig. 7A), suggesting impairments with the regulation of

$291 \mathrm{XCl}$ escape on the Xi in SLE. In sum, we identified a novel set of X-linked genes whose

292 expression is altered in SLE patient activated B cells, and the majority of these genes

293 should be subject to XCl silencing, reflecting aberrant gene regulation on both Xs.

294 One possible mechanism for aberrant XIST RNA localization in SLE patient activated B

295 cells could result from impairments with nuclear proteins that bind XIST RNA. The XIST

296 RNA Interactome consists of $\sim 275$ proteins $^{41-44}$, and we have previously reported that

297 two XIST RNA binding proteins, YY1 and hnRNP-U, are required for localization of XIST

298 RNA and heterochromatin marks to the Xi in lymphocytes ${ }^{31,35}$. Notably, SLE patient T

299 cells have altered expression of XIST RNA Interactome genes ${ }^{32}$. Thus, we asked

300 whether the expression of genes encoding XIST RNA binding proteins was also

301 abnormal in the activated B cells from SLE patients. We found that 80 XIST RNA

302 binding protein genes were differentially expressed in activated B cells, and the majority

303 of these genes (59/80; 74\%) were downregulated (Fig. 7C, Supplemental Table 5).

304 These genes function in cell metabolism/cell growth (genes in green), nuclear

305 matrix/nuclear envelope/transport (blue, but also includes $L B R$ and $h n R N P K$ ), and 
306 chromatin regulation (orange) (Fig. 7C). Downregulated genes LBR, $h n R N P K$, and 307 GLIPR2 (pink) also exhibited altered gene expression among SLE T cells relative to

308 healthy controls ${ }^{32}$. Taken together, the XIST RNA Interactome is dysregulated in 309 activated B cells from SLE patients, and may be responsible for mislocalization of XIST

$310 \mathrm{RNA}$ and heterochromatin modifications from the $\mathrm{Xi}$, resulting in aberrant $\mathrm{XCl}$

311 maintenance. 
317 B cells contribute to the pathogenesis of SLE, an autoimmune disease that 318 predominantly affects women. Here we sought to investigate the genetic basis for the 319 female-bias of SLE, focusing first on how $\mathrm{XCl}$ is maintained across distinct $\mathrm{B}$ cell 320 subsets in healthy individuals, and then determining if $\mathrm{XCl}$ maintenance and $\mathrm{X}$-linked 321 genes expression are affected in SLE. We discovered a diverse enrichment of 322 epigenetic modifications at the $\mathrm{Xi}$ across activated human B cell subsets, and found B 323 cell-specific patterns of $\mathrm{XCl}$ escape in healthy adults, including the escape of important 324 immune-related genes. Our profiling of pediatric and adult SLE patient B cells revealed 325 significant impairments with XIST RNA and H2AK119Ub enrichment on the Xi 326 irrespective of disease activity, and aberrant expression of X-linked genes. Together, 327 our results suggest that facultative chromatin of the $\mathrm{Xi}$ is relaxed in healthy $\mathrm{B}$ cells, 328 thereby altering $\mathrm{XCl}$ maintenance and permitting gene-specific escape from $\mathrm{XCI}$. SLE 329 disease further impacts the heterochromatic composition of this chromosome, resulting 330 in abnormal gene expression changes across the $\mathrm{X}$. Our epigenetic profiling of the $\mathrm{Xi}$ in 331 healthy and SLE B cells provides a foundation for future studies investigating the 332 molecular mechanisms of XIST RNA and heterochromatin mark localization and 333 spreading across the $\mathrm{Xi}$, and how these mechanisms become altered in SLE.

335 All four human B cell subsets assessed - naïve B, classical memory B, plasma cells, 336 and ABCs - are missing XIST RNA and the heterochromatic modification H2AK119Ub 337 on the Xi (Figure 1). Unexpectedly, our analyses show that unlike naïve B cells, ABCs, 
338 and plasma cells, memory B cells have XIST RNA transcripts dispersed across the 339 nucleus, yet still lack H2AK119Ub foci on the Xi (Figure 1C, 1D). At present, the 340 significance of dispersed XIST RNA transcripts in memory B cells is unknown. However,

341 it is likely to impact gene expression on the $\mathrm{Xi}$ as ex vivo $\mathrm{YY} 1$ deletion generates similar

342 Type III dispersed patterns with altered expression of $\sim 70$ X-linked genes ${ }^{35}$. The XIST

343 gene is expressed across all resting human B cell subsets (Figure 1E), thus

344 transcriptional changes do not account for the absence of XIST RNA transcripts on the

$345 \mathrm{Xi}$ in circulating human $\mathrm{B}$ cells. XIST RNA and the heterochromatin modifications $346 \mathrm{H} 2 \mathrm{AK} 119 \mathrm{Ub}, \mathrm{H} 3 \mathrm{~K} 27 \mathrm{me}$, and low levels of macroH2A returned to the $\mathrm{Xi}$ when using

347 CpG to activate human naïve B cells (Figure 2, 3, Supplemental S2). As for mouse 348 naïve B cells, XIST RNA and heterochromatin marks return to the Xi in human naïve B 349 cells before the first cell division ${ }^{35}$, with peak enrichment at day 2 post-stimulation and 350 prior to cell division ${ }^{45}$. We propose the Xi chromatin in circulating human B cell subsets 351 is more relaxed compared to somatic cells, in which XIST RNA and heterochromatin

352 marks are localized to the $\mathrm{Xi}$, and this may allow additional $\mathrm{X}$-linked genes to escape 353 transcriptional silencing. However, as our cytogenetic RNA FISH and IF analyses lack 354 resolution at the gene-level, it will be important to determine allele-specific enrichment 355 of silent and active histone modifications at genes exhibiting cell-specific $\mathrm{XCl}$ escape 356 and silencing across B cell subsets, and to further assess if such modifications are 357 altered upon activation.

359 Single-cell RNAseq profiling of four human B cell subsets from a healthy female 360 individual reveals cell-type specific $\mathrm{XCl}$ escape in naïve B cells, memory B cells, 
361 plasmablasts, and transitional B cells. The percentage of biallelically expressed X-linked

362 genes increase with B cell differentiation (Supplemental Table 3), which may reflect a

363 requirement for higher dosage of X-linked genes for proper function of memory B cells

364 and plasmablasts. For example, LAMP2, a lysosomal protein important for autophagy

365 and intracellular antigen presentation, is monoallelically expressed in transitional and

366 naïve B cells, yet biallelically expressed in memory B and plasmablasts (Fig. 4D). Such

367 results raise the intriguing possibility that biallelic expression of LAMP2 may increase

368 autophagy and antigen presentation, thereby contributing to enhanced immune

369 responses observed in females ${ }^{46}$. The X-linked gene IRAK1, responsible for IL1-

370 induced upregulation of NF-kappa B, is also biallelically expressed in memory B cells

371 and plasmablasts (Fig. 4D). Female neonates have higher levels of IRAK1 mRNA and

372 protein in cord blood and mononucleated cells compared to males ${ }^{47}$, potentially

373 contributing to reduced infection rates and female-specific immune advantages in

374 infants. However, these scRNAseq analyses are limited by the fact that the sample was

375 taken from one individual and does not contain in vivo stimulated B cells, which may

376 have distinct $\mathrm{XCl}$ escape profiles. As $\mathrm{XCl}$ escape exhibits individual variability when

377 comparing across human samples ${ }^{48}$, it will be important to repeat the allelic expression

378 profiling for human B cell subsets, especially activated B cells, using healthy female

379 individuals of different ages to determine which X-linked genes consistently escape

380 transcriptional silencing, and whether $\mathrm{XCl}$ escape increases with age.

382 Our investigations revealed abnormal $\mathrm{XCl}$ maintenance, as evidenced by reduced XIST

383 RNA and H2AK119Ub enrichment at the Xi, in both pediatric and adult SLE patient B 
384 cells, irrespective of disease activity. Perturbed XIST RNA and heterochromatin mark

385 localization to the $\mathrm{Xi}$ in lymphocytes is a feature of both human SLE and the analogous

386 lupus-like disease in the female-biased spontaneous mouse model NZB/W F1 ${ }^{32,49}$.

387 Activated SLE patient B cells exhibit altered expression of about 50 X-linked genes (Fig.

388 7A), and the majority of these genes were downregulated in SLE patients compared to

389 healthy controls. It is surprising that more than half of the downregulated X-linked genes

390 in SLE samples are putative XCl escape genes (Fig. 4 and previous studies in human

391 fibroblasts). The implications of reduced expression of these X-linked genes for B cell

392 function is unknown at this time. It is possible that aberrant XIST RNA localization and

393 reduced heterochromatic enrichment reflects alterations in the nuclear organization of

394 the $\mathrm{Xi}$ in SLE patient B cells. The $\mathrm{Xi}$, unlike the active $\mathrm{X}$ and autosomes, is organized

395 into two "megadomains" separated by a boundary region near the microsatellite repeat

$396 D x z 4^{50,51}$. During $X C l$ initiation, Xist RNA plays an important structural role for

397 configuring the $\mathrm{Xi}$ territory, and Xist deletion impairs megadomain formation ${ }^{52}$. XIST

398 RNA mislocalization in SLE patient B cells may reflect impairments to the Xi nuclear

399 territory, possibly resulting in abnormal gene silencing of some X-linked genes.

400

401 While altered X-linked gene expression can clearly impact SLE progression, our studies

402 cannot determine if abnormal $\mathrm{XCl}$ maintenance causes SLE disease, or is instead a

403 consequence of the disease. To date, there is no evidence that changes to the

404 extracellular environment can influence XCl maintenance in somatic cells. However, the

405 nuclear pore complex NUP43 and lamin B receptor (LBR), which are Xist RNA binding

406 proteins, were downregulated in SLE patient B cells (Fig. 7C). This may contribute to 
407 aberrant organization of the Xi nuclear territory in SLE patient B cells, as LBR protein 408 directly binds Xist RNA and this interaction is necessary for tethering the Xi to the

409 nuclear lamina and gene silencing ${ }^{53}$. While it is currently unclear if the inflammatory

410 environment of SLE affects nuclear architecture, it is tempting to speculate that

411 inflammatory cytokines or type I interferons, which are highly elevated in SLE patients

412 experiencing disease flares, may perturb $\mathrm{XCl}$ maintenance in $\mathrm{B}$ cells, resulting in altered

413 X-linked gene expression. Future studies to determine whether extrinsic factors can

414 influence $\mathrm{XCl}$ maintenance in lymphocytes will certainly reveal exciting new insights into

415 genetic and epigenetic factors responsible for sex-biased autoimmune disease. 
METHODS

Human B cell samples from healthy donors and SLE patients

422 Fresh and frozen PBMCs from adult healthy female donors were obtained from the

423 Penn Pathology BioResource core facility at the Perelman School of Medicine,

424 University of Pennsylvania. For comparative study of pediatric SLE patients (SLEDAI

425 score $=0$ ) and age-matched healthy controls, we recruited patients from the Children's

426 Hospital of Philadelphia (CHOP). Approximately 15-20 mL of blood were collected from

427 each individual, stored on ice, then immediately processed for PBMC isolation. PBMCs

428 were separated from whole blood by density gradient centrifugation technique using

429 Lymphoprep media (cat \# 07851, STEMCELL technologies, Cambridge, MA, USA).

430 PBMCs from CHOP patients were either frozen or used to isolate $B$ cells immediately,

431 and we did not observe any effect of freeze/thaw on XIST RNA localization patterns.

432 PBMCs were frozen in fetal bovine serum containing 7\%-10\% DMSO. We also obtained

433 frozen PBMC samples from adult SLE patients (SLEDAI score: 0-20; age 18-63 yrs.)

434 and age-matched healthy controls from the Benaroya Research Institute, Seattle,

435 Washington. The acquisition of blood samples from pediatric SLE patients and healthy

436 controls from CHOP was approved by the IRB at CHOP; acquisition of blood from adult

437 SLE patients and healthy controls from the Benaroya Institute was approved by the IRB

438 at the Benaroya Institute. Written informed consent was received from participants prior

439 to inclusion in both studies.

Sorting and culture of human B cells

443 Frozen PBMCs were quickly thawed and washed twice with RPMI media containing 444 FBS. CD19 ${ }^{+}$CD27 $7^{-}$naïve B cells and $\mathrm{CD}_{19}{ }^{+} \mathrm{CD} 27^{+}$memory B cells were isolated from 445 PBMCs using the Easysep human memory B cell isolation kit, according to the 446 manufacture's instruction (17864, STEMCELL Technologies, Cambridge, MA, USA). $447 \mathrm{CD}_{19} \mathrm{CD}^{\mathrm{C}} 138^{+}$plasma cells were isolated from PBMCs by positive selection using biotin 448 conjugated CD138 antibody (352322, Biolegend, San Diego, CA, USA) and Easysep 
449 release human biotin positive selection cocktail (17653, STEMCELL Technologies). $450 \mathrm{CD}_{19}^{+} \mathrm{CD} 11 \mathrm{c}^{+} \mathrm{B}$ cells (ABCs) were isolated from PBMCs using a two-step procedure. 451 First, total B cells were isolated from PBMCs by negative selection using the human B 452 Cell Isolation Kit II (130-091-151, Miltenyi Biotech, Cambridge, MA, USA). ABCs were 453 isolated from purified total B cells by CD11c positive selection using a biotin conjugated 454 CD11c antibody (301612, Biolegend) and anti-biotin magnetic beads. B cells were 455 cultured in X-VIVO ${ }^{\mathrm{TM}} 15$ media (04-744Q, Lonza, Walkersville, MD, USA) with penicillin456 streptomycin (100 units/mL) and activated using $3 \mu \mathrm{M} \mathrm{CpG} \mathrm{(ODN} \mathrm{7909)} \mathrm{(tIrl-2006-1,}$ 457 Invivogen, San Diego, CA, USA). Cells were cultured in $200 \mu$ medium for 1-8 days 458 using round bottom 96-well plates. B cell stimulation was determined by staining for the 459 activation marker CD86, which was quantified using flow cytometry.

Flow cytometry profiling of naïve $B$ cells $\left(C D 19^{+} C D 27^{-}\right)$from SLE and HC subjects

We used multicolor flow cytometry analysis to determine the subset distribution B cells in SLE and HC samples ${ }^{54,55}$. B cell subsets were phenotyped as follows: naïve B cells $\left(\mathrm{CD} 19^{+} \mathrm{CD} 10^{-} \mathrm{CD} 21^{+} \mathrm{IgD}^{+}\right)$, transitional B cells $\left(\mathrm{CD} 19^{+} \mathrm{CD} 10^{+} \mathrm{CD} 38^{+}\right)$, memory $\mathrm{B}$ cells $\left(\mathrm{CD}_{19}{ }^{+} \mathrm{CD} 27^{+}\right)$, plasma $\mathrm{B}$ cells (CD19+CD138+), and ABCs (CD19 ${ }^{+} \mathrm{CD} 10^{-} \mathrm{CD} 21^{-}$

467 CD85j $^{+}$). Antibodies (and catalog numbers) used for flow cytometry were: FITC CD85j 468 (555942, BD Biosciences), Brilliant Violet 421 TM CD38 (303525, Biolegend), 469 Brilliant Violet $650^{\text {TM }}$ CD27 (302827, Biolegend), Brilliant Violet $785^{\text {TM }}$ CD19 470 (302239, Biolegend), APC/Cy7 CD3 (300425, Biolegend), APC/Cy7 CD14 471 (561709, BD Biosciences), APC/Cy7 CD16(561726, BD Biosciences), PE472 CF594 IgD (562540, BD Biosciences), PE/Cy7 CD21 (354911, Biolegend), 473 BV605 CD24 (311123, Biolegend), PECy5 CD10 (15-0106-41, Thermo Fisher) 474 and LIVE/DEAD ${ }^{\text {тм }}$ Fixable Aqua Dead Cell Stain Kit (L34965, Thermo Fisher).

Sequential XIST RNA FISH and immunofluorescence (IF)

478 Sequential XIST RNA fluorescence in situ hybridization (FISH) and IF was performed 479 using established protocols ${ }^{31,32}$. Briefly, cells were cytospun onto glass slides, then 
480 incubated in ice-cold cytoskeletal (CSK) buffer containing $0.5 \%$ Triton for 3 min, fixed in $4814 \%$ paraformaldehyde for $10 \mathrm{~min}$, and then dehydrated using an ethanol series. For 482 human XIST RNA FISH, we used two Cy3 labelled oligonucleotide probes which target 483 repetitive regions within XIST exons 1, 3 and $4^{31}$. Images were obtained using a Nikon 484 Eclipse microscope and were categorized by the type of XIST RNA localization patterns 485 as shown in Figure $1 \mathrm{~B}$ and as described previously ${ }^{31,33,35}$. For IF analyses, slides were 486 blocked for 30 min in blocking buffer (PBS with $0.2 \%$ Tween-20 and 5\% BSA) and then 487 incubated for 2 hours at room temperature with respective primary antibodies (at 488 dilutions of 1:100): H3K27me3 (39155, Active Motif); Ubiquityl-histone H2A Lys119 489 (8240, Cell Signaling); H3K4me3 (ab 213224, Abcam); MacroH2A1 (ab37264, Abcam). 490 Slides were incubated with the appropriate FITC conjugated secondary antibody for 1 491 hour at room temperature, then imaged using a fluorescence microscope.

493 quantitative RT-PCR

For RT-QPCR, cDNA was synthesized from $1 \mu \mathrm{g}$ of total RNA using Verso cDNA synthesis kit (Ref \# AB-1453/A, Thermo Fisher, Waltham, MA, USA) and qPCR was performed using the QuantBio low ROX cyber green master mix (\# 017707, Quantabio, Beverly, MA, USA). RPL13A gene was used as an endogenous control to normalize gene expression. RT-QPCR data were analyzed using $\Delta \Delta \mathrm{Ct}$ method $^{5}$.

500 RPL13A, F: GCCATCGTGGCTAAACAGGTA， R: GTTGGTGTTCATCCGCTTGC; macroH2A1.1, F: GGCTTCACAGTCCTCTCCAC, R: GGTGAACGACAGCATCACTG; macroH2A1.2, F: GGCTTCACAGTCCTCTCCAC, R: GGATTGATTATGGCCTCCAC, 503 XIST 5' end: F: TTGCCCTACTAGCTCCTCGGAC, R: TTCTCCAGATAGCTGGCAACC; XIST 3' end: F: CTACAAGCAGTGCAGAGAGC, R: CTAAGACAAGACACAGACCAC.

507 RNA-seq analysis of activated B cells from SLE patients and controls 
511 from GEO dataset

GSE118254

512 (https://www.ncbi.nlm.nih.gov/geo/query/acc.cgi?acc=GSE118254).

Statistical

513 significance in gene expression between Healthy and SLE B cells was calculated using

514 a one-way ANOVA in R (alpha = 0.05). A Z score was calculated for each sample/gene

515 and heatmaps were generated using the gplots (heatmap.2) R package.

Allele specific gene expression analyses using single cell RNAseq data

519 Single cell RNAseq reads sequenced from individual naïve B cells, memory B cells,

520 plasmablasts and transitional B cells were retrieved from a previously published study

521 (Bioproject accession number PRJEB27270) ${ }^{38}$. In this study, a total of 117 cells, 522 consisting of 30 naive B cells, 30 memory B cells, 30 plasmablasts and 27 transitional B 523 cells, were sequenced from a single healthy woman. Public servers available through 524 the Galaxy web platform (usegalaxy.org) were used to analyze the data ${ }^{56}$. RNA-Seq 525 reads were mapped to human reference genome (version hg19) using Bowtie ${ }^{57,58}$. Next, 526 SNPs present in both $X$ chromosomes were detected from alignment file using the 527 FreeBayes variant detector tool (www.geneious.com) ${ }^{59}$ and SNPs were annotated using 528 the ANNOVAR tool ${ }^{60}$. SNPs with less than 10 reads were excluded from the 529 downstream analyses. For each SNP, if $\leq 90 \%$ of the reads carry the same SNP allele, 530 expression was considered biallelic, otherwise expression was considered monoallelic 531 for each gene. A gene was considered 'biallelic' in a cell-type subset if 2 or more cells 532 from that subset were considered 'biallelic' as described above; a gene was considered 533 "monoallelic" is 2 or more cells were biallelic and "uncalled" if no two cells were in 534 agreement or there was insufficient data to make a call.

Statistical analysis

538 Linear regression was performed using a simple linear regression in GraphPad Prism 8 539 of dependent variables (Xist RNA Cloud Type I-V) and independent variables (Date of 540 blood draw for PBMC sample, SLE disease duration, Anti-Nuclear Antibody Titer, 541 Systemic Lupus Erythematosus Disease Activity Index (SLEDAI) score and patient age 
542 at blood draw). Measurements of percent Type I-IV XIST RNA clouds were analyzed

543 using unpaired, two-tail t-tests, where significance is $p<0.05$.

Study approval

For pediatric SLE patients, protocols and informed consent forms were approved by the CHOP Institutional Review Board (CHOP IRB Protocol 14-011433). For the adult SLE patients, the studies were approved by the Benaroya Research Institute Institutional

550 Review Committee (IRB protocol number: 10059).

Figure Legends

Figure 1: XIST RNA signals are missing from the $X i$ in human $B$ cell populations.

(A) Cartoon representing each type of XIST RNA localization pattern observed in

556 human B cell subsets. (B) Sequential XIST RNA FISH (red) then immunofluorescence

557 detection (green) for H2AK119-ubiquitin (Ub) for naïve B cells, memory B cells, age-

558 associated B cells (ABCs), and plasma cells from healthy PBMCs. (C) Quantification of

559 XIST RNA localization patterns from each B cell subset. Number of nuclei counted is

560 shown above each sample at the top of the graph. Statistical significance for each XIST

561 RNA localization pattern determined using one-way ANOVA; $p$ values for each pattern

562 shown. (D) Quantification of H2AK119Ub foci in human B cell subsets. Number of nuclei

563 counted is shown above each sample at the top of the graph. (E) XIST RNA reads for

564 naïve $B, A B C s$, and memory $B$ cells from a previously published RNAseq dataset ${ }^{34}$.

Figure 2: Timing for XIST RNA localization to the $\mathrm{Xi}$ during human $\mathrm{B}$ cell

567 stimulation, for naïve and memory B cells. (A) Time course analysis for XIST RNA

568 FISH to monitor XIST RNA localization changes after B cell stimulation using CpG, over 
5697 days. (B) CD86+ staining of in vitro activated B cells (day 1, day 2) to measure

570 efficiency of in vitro stimulation. (C) Quantification of XIST RNA localization patterns for

571 in vitro stimulated naïve B cells over 7 days in culture. Number of nuclei counted is

572 shown above each sample at the top of the graph. Statistical significance determined

573 using t-test comparing day 0 to day 2, for each type of XIST RNA localization pattern.

574 (D) XIST RNA FISH for in vitro stimulated memory B cells using CpG, over 3 days. (E)

575 Quantification of XIST RNA localization patterns for in vitro activated memory B cells

576 over 3 days. Number of nuclei counted is shown above each sample at the top of the

577 graph. Statistical significance determined using t-test comparing day 0 to day 3 , for

578 each type of XIST RNA localization pattern.

579

Figure 3: Co-localization of XIST RNA and heterochromatin marks H2AK119Ub

581 and H3K27me for in vitro activated human B cells. Sequential XIST RNA FISH (red)

582 followed by immunofluorescence detection (green) for (A) H2AK119Ub and (B)

583 H3K27me3. Representative images (showing the same field) are shown. Quantification

584 of co-localization patterns for XIST RNA and each heterochromatin mark at the Xi. Co-

585 localization of XIST RNA (Types I, II) and IF focus (blue bars), XIST RNA signals alone

586 (Type III; green), nuclei without either signal (purple), or IF focus (orange). Number of 587 nuclei counted is above each sample.

589 Figure 4: Biallelic expression of X-linked genes in human B cell populations. (A)

590 Schematic of the bioinformatics analysis pipeline to identify $\mathrm{XCl}$ escape genes in 591 circulating memory B cells, naïve B cells, transitional B cells, and plamsablasts. (B) 
592 Heatmap for all X-linked genes with detectable expression across the four human B cell

593 subsets. Each individual cell is a column. Light blue indicates biallelic expression (Minor

594 Allele Frequency (MAF) $\geq 0.1$ for $>50 \%$ of SNPs per gene), dark blue indicates

595 monoallelic expression, and white is undetectable expression. Gene lists for individual

596 SNPs in each cell (across B cell populations) are found in Supplemental Table 1, and

597 complete list of all expressed X-linked genes is in Supplemental Table 2. (C) Expression

598 of X-linked immunity-related genes across all four B cell populations. Individual cells for

599 a particular B cell subset shown in columns; monoallelic expression (dark blue); biallelic

600 expression (light blue). Supplemental Table 3 contains gene lists for each B cell subset.

601 (D) Allelic expression summary for the X-linked immunity-related genes, either

602 monoallelic (dark blue) or biallelic (light blue) across human B cell subsets. A gene was

603 considered biallelic for a particular B cell subset if 2 or more cells within that group were

604 biallelic. A gene was considered monoallelic for a B cell subset if 2 or more cells within 605 that subset were monoallelic. Supplemental Table 4 contains complete list of X-linked 606 immunity-related genes that were expressed in each B cell subset, along with allelic 607 expression information.

609 Figure 5: Peripheral B cells from pediatric SLE patients have mislocalized XIST

RNA patterns and lack H2AK119Ub foci at the Xi. (A) Representative XIST RNA

611 FISH images from in vitro activated B cells (cultured 2 days) from one pediatric SLE 612 patient (right) and a healthy age-matched control (left). (B) Quantification of Type I (left),

613 Type II (center) and Type IV (right) XIST RNA localization patterns for in vitro activated 614 B cells from pediatric SLE patients (red) and healthy controls (blue). Error bars denote 
615 mean +/-SD, and statistical significance was determined using two-tailed unpaired t-test.

616 (C) Quantification of H2AK119Ub foci for in vitro activated B cells from pediatric SLE

617 patients and healthy control samples. Number of nuclei counted is above each sample;

618 statistical significance comparing SLE to healthy controls was determined using two-

619 tailed unpaired t-test. (D) Quantification of XIST RNA localization patterns for in vitro 620 activated classical memory B cells, cultured for 3 days with CpG. Number of nuclei

621 counted is above each sample; statistical significance comparing SLE to healthy

622 controls was determined using two-tailed unpaired t-test for each pattern of XIST RNA

623 localization.

625 Figure 6: Peripheral B cells from adult SLE patients have mislocalized XIST RNA

626 patterns and reduced H2AK119Ub foci at the Xi. (A) Representative XIST RNA

627 FISH images from in vitro activated B cells (cultured 2 days) from one adult SLE patient

628 (right) and a healthy age-matched control (left). (B) Quantification of Type I (left), Type II

629 (center) and Type IV (right) XIST RNA localization patterns for in vitro activated B cells

630 from adult SLE patients (red) and healthy controls (blue). Error bars denote mean +/-

631 SD, and statistical significance was determined using two-tailed unpaired t-test. (C)

632 Quantification of co-localization patterns for XIST RNA and H2AK119Ub at the Xi, for in

633 vitro activated B cells from adult SLE patients and healthy control samples. Co-

634 localization of XIST RNA (Types I, II) and IF focus (blue bars), XIST RNA signals alone

635 (Type III; green), nuclei without either signal (purple), or IF focus (orange). Number of

636 nuclei counted is above each sample; statistical significance comparing SLE to healthy 
637 controls was determined using two-tailed unpaired t-test for each pattern of XIST RNA

638 localization.

640 Figure 7: X-linked gene expression and XIST RNA Interactome genes are altered

641 in SLE patient activated B cells. (A) X-linked Differentially Expressed Genes (DEGs;

64253 genes) in adult healthy controls (6 female samples) and SLE patient (7 female 643 samples) activated B cells in circulation. Color gradient represents row Z-scores for 644 each gene. Gene symbols in color denote $\mathrm{XCl}$ escape: orange are immunity-related 645 genes that may escape in activated B cells; green are known XCl escape genes in other 646 somatic cells; blue are putative $\mathrm{XCl}$ escape based on Supplemental Table 3. Note that

647 all 4 genes in orange exhibited $\mathrm{XCl}$ escape in at least $1 \mathrm{~B}$ cell subset in Supplemental 648 Table 3, and are denoted with (e). (B) Volcano plot showing X-linked DEGs in activated 649 B cells. Genes significantly upregulated in SLE patients are in red; genes significantly 650 downregulated in SLE are in blue $(p<0.05)$. (C) XIST RNA binding protein genes that 651 are differentially expressed in activated B cells from SLE patients and healthy controls. 652 Nuclear matrix/nuclear envelop genes in blue; cell metabolism/cell growth genes in 653 green; chromatin regulators in orange; XIST RNA binding protein genes whose 654 expression was also altered in SLE patient T cells in pink.

656 Supplemental Figure 1: Steady-state XIST RNA transcript levels in human naïve 657 and in vitro stimulated B cells. Primer sets for 5' XIST (spanning exons 1 and 3) and 658 3' XIST (spanning exons 5 and 6) were used for qRT-PCR analysis of XIST RNA in 659 naïve (blue) and in vitro stimulated (orange) B cells. Relative fold change is shown, with 
660 values normalized to naïve B cells (set as 1). Standard error of the mean is shown with

661 error bars.

663 Supplemental Figure 2: macroH2A is not uniformly enriched on the Xi in activated

664 B cells in healthy donors. (A) Sequential XIST RNA FISH (red) followed by

665 immunofluorescence detection of histone variant macroH2A (green). Representative

666 field is shown, and arrowheads indicate macroH2A foci that co-localize with XIST RNA

667 signal. (B) Quantification of XIST RNA localization patterns that co-localize with

668 macroH2A foci. Number of nuclei counted is above each sample. (C) qRT-PCR

669 analyses of macroH2A1.1 and macroH2A1.2 transcripts in naïve and in vitro stimulated

670 (day 2) B cells (left). qRT-PCR analyses of both macroH2A1 variants for naïve and

671 stimulated B cells compared to 293T, a human embryonic kidney fibroblast cell line

672 (right). Statistical significance determined using one-way ANOVA across three cell types

673 for each variant.

674

675 Supplemental Figure 3: Sequential XIST RNA FISH and IF detection of the active

676 chromatin modification H3K4me3. (A) Representative field image for XIST RNA FISH

677 (red) and sequential IF for H3K4me3 (green). The arrowheads indicate H3K4me3

678 'holes' that overlap XIST RNA signals. (B) Quantification of XIST RNA localization

679 patterns and H3K4me3 'holes'. Number of nuclei counted is above each sample.

680

681 Supplemental Figure 4: XIST RNA localization patterns for naïve and in vitro 682 stimulated B cells (day 2) from pediatric SLE patients and healthy age-matched 
683 controls. (A) Quantification of XIST RNA localization patterns for naïve B cells. Number

684 of nuclei counted is above each sample. (B) Quantification of XIST RNA localization

685 patterns for in vitro stimulated (day 2) B cells using CpG. Number of nuclei counted is

686 above each sample. (C) Representative flow cytometry analysis for CD86 staining,

687 using unstained cells, naïve B (unstimulated), and day 2 stimulated B cells for a healthy

688 control sample and one pediatric SLE patient sample (SLE 17). (D) Quantification of 689 XIST RNA localization patterns for in vitro stimulated memory B cells from pediatric SLE

690 patients and healthy controls. Number of nuclei counted is above each sample.

691 Statistical significance determined using two-tailed test with unequal variance, 692 comparing SLE to healthy controls. P values for each localization pattern of XIST RNA 693 are shown.

695 Supplemental Figure 5: Flow cytometry analyses of B cell populations from 696 PBMCs of adult and pediatric SLE patients and age-matched healthy controls. (A)

697 Typical gating strategy for B cell subsets from patient PBMCs recovered post-thaw, 698 following $B$ cell isolation and selection for CD27- cells. (B) CD27- B cell population 699 percentages for healthy control $(\mathrm{HC})$ and SLE patients. Average percentages are shown 700 in bold, at the bottom of each group. Pediatric samples are denoted as "P2". Statistical 701 significance determined using two-tailed t-tests comparing healthy controls to SLE, for 702 each B cell subset. Only ABCs were significantly different among SLE and HC 703 samples, yet comprise less than $2 \%$ of total B cells. 
Supplemental Figure 6: XIST RNA localization patterns for in vitro stimulated B cells (day 2) from adult SLE patients and healthy age-matched controls. Number of nuclei counted is above each sample. Statistical significance was determined using two-

708 tailed unpaired t-test for each pattern of XIST RNA localization, comparing SLE to 709 healthy controls. SLE samples 580-253 and 342-184 are male individuals, and are 710 included as negative controls. autoimmunity comorbidities and medications. Bar graphs show percent of activated

715 without thyroid disease or Sjogren's Syndrome. Red, green, and blue bar graph pairs represent significant differences between group means. Bar graphs show percent of

717 activated B cells with XIST RNA localization patterns Type I-IV (A-D) for adult SLE 718 patients. The paired bar graphs consist of patients taking (left) or not taking (right) the 719 designated medication. Blue bar graph pairs represent significant differences between 720 group means; statistical significance determined without correction for multiple 721 comparisons with alpha $=0.05$. Each row was analyzed individually, without assuming a

722 consistent SD. HCQ: hydroxychloroquine; mycophen. mofetil: mycophenolate mofetil.

723 (E) SLE patients taking HCQ have significantly higher percentages of Type I XIST RNA

724 localization patterns. Dotplot showing percentages of B cells for groups of HC and SLE

725 patients for each type of XIST RNA localization pattern. Black circles represent

726 individuals not taking HCQ; aqua triangles are SLE patients taking HCQ. Horizontal

727 bars show median values of patients taking HCQ (aqua) or other medications (black). 
Only Type I XIST RNA patterns were statistically significant among SLE patients for

$729 \mathrm{HCQ}$ treatment. Statistical significance determined using two-tailed t test.

\section{Supplemental Figure 8: Linear regression analyses of SLE patient disease}

parameters. (A) SLE disease parameters (ANA Titer, SLEDAI score, disease duration),

733 patient age, and sample draw date were correlated with each XIST RNA localization

734 pattern for in vitro activated adult SLE B cell samples. (B) Correlation between age and

735

736

737

738

739

740

741

742

743

744

745

746

747

748

749

750

751

752

753

754

755

756

XIST RNA localization patterns for SLE patients (left) and healthy controls (right). XIST

RNA Type IV patterns increased with age for SLE patients; there were no significant correlations between age and XIST RNA localization patterns for healthy controls.

\section{Acknowledgements}

We would like to thank $\mathrm{C}$. Berry for statistical consultation for correlation analyses; N. Jiwrajka and L. King for assistance with editing of the manuscript, and all members of the Anguera lab for helpful discussions. This research was supported by a University Research Foundation grant, a American Chemical Society grant, a McCabe Foundation grant), NIH R21 Al124084, NICHD 5K12 HD085848-03, NIH R01 Al 134834, DOD grant LR170055: W81XWH-18-1-06 (to MCA); and NIH 1F32Al154797 to SP.

\section{Author contributions}

S. Pyfrom performed the bioinformatic analyses for single-cell RNAseq data sets, the bioinformatic analyses using human activated B cell RNAseq data sets, and the linear regression analyses for XIST RNA localization and comorbidities, and made corresponding supplementary tables and figures. B. Paneru performed the human B cell isolation and in vitro culture, the XIST RNA FISH and IF experiments on human B cell subsets, and quantified the localization patterns for SLE patients and controls. J. Knoxx and B. Paneru performed the flow cytometry and FACS isolation experiments. MCA 
made figures $1,2,3,5,6$ in main text and supplementary figures $\mathrm{S} 1, \mathrm{~S} 2, \mathrm{~S} 3, \mathrm{~S} 4, \mathrm{~S} 5$,

S6, S6. MCA and S. Pyfrom wrote the manuscript.

1 Wei, C. et al. A new population of cells lacking expression of CD27 represents a

\title{
Competing interests
}

\author{
None to declare.
}

\section{REFERENCES} notable component of the B cell memory compartment in systemic lupus erythematosus. J Immunol 178, 6624-6633, doi:10.4049/jimmunol.178.10.6624 (2007).

2 Nicholas, M. W. et al. A novel subset of memory B cells is enriched in autoreactivity and correlates with adverse outcomes in SLE. Clin Immunol 126, 189-201, doi:10.1016/j.clim.2007.10.004 (2008).

3 Tipton, C. M. et al. Diversity, cellular origin and autoreactivity of antibodysecreting cell population expansions in acute systemic lupus erythematosus. Nat Immunol 16, 755-765, doi:10.1038/ni.3175 (2015).

4 Rubtsov, A. V., Rubtsova, K., Kappler, J. W. \& Marrack, P. TLR7 drives accumulation of $\mathrm{ABC}$ and autoantibody production in autoimmune-prone mice. Immunol Res 55, 210-216, doi:10.1007/s12026-012-8365-8 (2013).

5 Hao, Y., O'Neill, P., Naradikian, M. S., Scholz, J. L. \& Cancro, M. P. A B-cell subset uniquely responsive to innate stimuli accumulates in aged mice. Blood 118, 1294-1304, doi:10.1182/blood-2011-01-330530 (2011).

6 Libert, C., Dejager, L. \& Pinheiro, I. The X chromosome in immune functions: when a chromosome makes the difference. Nat Rev Immunol 10, 594-604, doi:10.1038/nri2815 (2010).

7 Liu, K. et al. X Chromosome Dose and Sex Bias in Autoimmune Diseases: Increased 47,XXX in Systemic Lupus Erythematosus and Sjogren's Syndrome. Arthritis Rheumatol, doi:10.1002/art.39560 (2015).

8 Ross, M. T. et al. The DNA sequence of the human X chromosome. Nature 434, 325-337, doi:10.1038/nature03440 (2005).

9 Bianchi, I., Lleo, A., Gershwin, M. E. \& Invernizzi, P. The X chromosome and immune associated genes. J Autoimmun 38, J187-192, doi:10.1016/j.jaut.2011.11.012 (2012).

10 Garcia-Ortiz, H. et al. Association of TLR7 copy number variation with susceptibility to childhood-onset systemic lupus erythematosus in Mexican

801 population. Ann Rheum Dis 69, 1861-1865, doi:10.1136/ard.2009.124313 (2010). 
11 Desai-Mehta, A., Lu, L., Ramsey-Goldman, R. \& Datta, S. K. Hyperexpression of CD40 ligand by $B$ and $T$ cells in human lupus and its role in pathogenic autoantibody production. J Clin Invest 97, 2063-2073, doi:10.1172/JCI118643 (1996).

12 Vadasz, Z. et al. The Expansion of CD25 high IL-10 high FoxP3 high B Regulatory Cells Is in Association with SLE Disease Activity. J Immunol Res 2015, 254245, doi:10.1155/2015/254245 (2015).

13 Kong, W. et al. Increased expression of Bruton's tyrosine kinase in peripheral blood is associated with lupus nephritis. Clin Rheumatol 37, 43-49, doi:10.1007/s10067-017-3717-3 (2018).

14 Pisitkun, $\mathrm{P}$. et al. Autoreactive $\mathrm{B}$ cell responses to RNA-related antigens due to TLR7 gene duplication. Science 312, 1669-1672, doi:10.1126/science.1124978 (2006).

15 Subramanian, S. et al. A TIr7 translocation accelerates systemic autoimmunity in murine lupus. Proc Natl Acad Sci U S A 103, 9970-9975, doi:10.1073/pnas.0603912103 (2006).

16 Fairhurst, A. M. et al. Yaa autoimmune phenotypes are conferred by overexpression of TLR7. Eur J Immunol 38, 1971-1978, doi:10.1002/eji.200838138 (2008).

17 Hwang, S. H. et al. B cell TLR7 expression drives anti-RNA autoantibody production and exacerbates disease in systemic lupus erythematosus-prone mice. J Immunol 189, 5786-5796, doi:10.4049/jimmunol.1202195 (2012).

18 Kil, L. P. et al. Btk levels set the threshold for B-cell activation and negative selection of autoreactive B cells in mice. Blood 119, 3744-3756,

doi:10.1182/blood-2011-12-397919 (2012).

19 Corneth, O. B. et al. Enhanced Expression of Bruton's Tyrosine Kinase in B Cells Drives Systemic Autoimmunity by Disrupting T Cell Homeostasis. J Immunol 197, 58-67, doi:10.4049/jimmunol.1600208 (2016).

20 Lyon, M. F. Gene action in the X-chromosome of the mouse (Mus musculus L.). Nature 190, 372-373 (1961).

21 Payer, B. \& Lee, J. T. X chromosome dosage compensation: how mammals keep the balance. Annu Rev Genet 42, 733-772, doi:10.1146/annurev.genet.42.110807.091711 (2008).

22 Plath, K. et al. Role of histone H3 lysine 27 methylation in X inactivation. Science 300, 131-135, doi:10.1126/science.1084274

1084274 [pii] (2003).

23 Silva, J. et al. Establishment of histone h3 methylation on the inactive $X$ chromosome requires transient recruitment of Eed-Enx1 polycomb group

845

24 Cotton, A. M. et al. Landscape of DNA methylation on the X chromosome reflects CpG density, functional chromatin state and X-chromosome inactivation. Hum Mol Genet 24, 1528-1539, doi:10.1093/hmg/ddu564 (2015).

25 Sharp, A. J. et al. DNA methylation profiles of human active and inactive $X$ chromosomes. Genome Res 21, 1592-1600, doi:10.1101/gr.112680.110 (2011). 
84626 Brockdorff, N. et al. Conservation of position and exclusive expression of mouse Xist from the inactive $X$ chromosome. Nature 351, 329-331, doi:10.1038/351329a0 (1991).

27 Brown, C. J. et al. A gene from the region of the human $X$ inactivation centre is expressed exclusively from the inactive $X$ chromosome. Nature 349, 38-44, doi:10.1038/349038a0 (1991). Requirement for Xist in $\mathrm{X}$ chromosome inactivation. Nature 379, 131-137, doi:10.1038/379131a0 (1996).

29 Carrel, L. \& Willard, H. F. X-inactivation profile reveals extensive variability in Xlinked gene expression in females. Nature 434, 400-404, doi:10.1038/nature03479 (2005).

30 Balaton, B. P., Cotton, A. M. \& Brown, C. J. Derivation of consensus inactivation status for X-linked genes from genome-wide studies. Biol Sex Differ 6, 35, doi:10.1186/s13293-015-0053-7 (2015).

31 Wang, J. et al. Unusual maintenance of $X$ chromosome inactivation predisposes

863

864 female lymphocytes for increased expression from the inactive X. Proc Natl Acad Sci U S A, doi:10.1073/pnas.1520113113 (2016).

32 Syrett, C. M. et al. Altered X-chromosome inactivation in T cells may promote sex-biased autoimmune diseases. JCI Insight 4, doi:10.1172/jci.insight.126751 (2019).

33 Syrett, C. M. et al. Diversity of Epigenetic Features of the Inactive XChromosome in NK Cells, Dendritic Cells, and Macrophages. Front Immunol 9, 3087, doi:10.3389/fimmu.2018.03087 (2018).

34 Wang, S. et al. IL-21 drives expansion and plasma cell differentiation of autoreactive CD11c(hi)T-bet(+) B cells in SLE. Nat Commun 9, 1758, doi:10.1038/s41467-018-03750-7 (2018).

35 Syrett, C. M. et al. Loss of Xist RNA from the inactive X during B cell development is restored in a dynamic YY1-dependent two-step process in activated B cells. PLoS Genet 13, e1007050, doi:10.1371/journal.pgen.1007050 (2017).

36 Ridings-Figueroa, $\mathrm{R}$. et al. The nuclear matrix protein $\mathrm{ClZ1}$ facilitates localization of Xist RNA to the inactive X-chromosome territory. Genes Dev 31, 876-888, doi:10.1101/gad.295907.117 (2017).

37 Lessing, D., Anguera, M. C. \& Lee, J. T. X Chromosome Inactivation and Epigenetic Responses to Cellular Reprogramming. Annu Rev Genomics Hum Genet, doi:10.1146/annurev-genom-091212-153530 (2013).

38 Rizzetto, S. et al. B-cell receptor reconstruction from single-cell RNA-seq with VDJPuzzle. Bioinformatics 34, 2846-2847, doi:10.1093/bioinformatics/bty203 (2018).

885

886

887

888

39 Souyris, M. et al. TLR7 escapes X chromosome inactivation in immune cells. Sci Immunol 3, doi:10.1126/sciimmunol.aap8855 (2018).

889

40 Scharer, C. D. et al. Epigenetic programming underpins B cell dysfunction in human SLE. Nat Immunol 20, 1071-1082, doi:10.1038/s41590-019-0419-9

890 (2019). 
89141 Chu, C. et al. Systematic discovery of Xist RNA binding proteins. Cell 161, 404416, doi:10.1016/j.cell.2015.03.025 (2015).

89342 McHugh, C. A. et al. The Xist IncRNA interacts directly with SHARP to silence transcription through HDAC3. Nature 521, 232-236, doi:10.1038/nature14443 (2015).

43 Minajigi, A. et al. Chromosomes. A comprehensive Xist interactome reveals cohesin repulsion and an RNA-directed chromosome conformation. Science 349, doi:10.1126/science.aab2276 (2015).

900

901

902

903

904

905

906

46 Klein, S. L. \& Flanagan, K. L. Sex differences in immune responses. Nat Rev

44 Monfort, A. et al. Identification of Spen as a Crucial Factor for Xist Function through Forward Genetic Screening in Haploid Embryonic Stem Cells. Cell Rep 12, 554-561, doi:10.1016/j.celrep.2015.06.067 (2015).

45 Tangye, S. G., Avery, D. T., Deenick, E. K. \& Hodgkin, P. D. Intrinsic differences in the proliferation of naive and memory human $B$ cells as a mechanism for enhanced secondary immune responses. J Immunol 170, 686-694, doi:10.4049/jimmunol.170.2.686 (2003).

908 Immunol 16, 626-638, doi:10.1038/nri.2016.90 (2016).

47 O'Driscoll, D. N. et al. Expression of X-linked Toll-like receptor 4 signaling genes in female vs. male neonates. Pediatr Res 81, 831-837, doi:10.1038/pr.2017.2 (2017).

48 Tukiainen, $T$. et al. Landscape of $X$ chromosome inactivation across human tissues. Nature 550, 244-248, doi:10.1038/nature24265 (2017).

49 Syrett, C. M., Sierra, I., Beethem, Z. T., Dubin, A. H. \& Anguera, M. C. Loss of epigenetic modifications on the inactive $X$ chromosome and sex-biased gene expression profiles in B cells from NZB/W F1 mice with lupus-like disease. $J$ Autoimmun, 102357, doi:10.1016/j.jaut.2019.102357 (2019).

Deng, X. et al. Bipartite structure of the inactive mouse X chromosome. Genome Biol 16, 152, doi:10.1186/s13059-015-0728-8 (2015).

51 Giorgetti, L. et al. Structural organization of the inactive X chromosome in the mouse. Nature 535, 575-579, doi:10.1038/nature18589 (2016).

52 Splinter, E. et al. The inactive $X$ chromosome adopts a unique three-dimensional conformation that is dependent on Xist RNA. Genes Dev 25, 1371-1383, doi:10.1101/gad.633311 (2011).

53 Chen, C. K. et al. Xist recruits the $\mathrm{X}$ chromosome to the nuclear lamina to enable chromosome-wide silencing. Science 354, 468-472, doi:10.1126/science.aae0047 (2016).

54 Knox, J. J. et al. T-bet+ B cells are induced by human viral infections and dominate the HIV gp140 response. JCI Insight 2, doi:10.1172/jci.insight.92943 (2017).

55 Johnson, J. L. et al. The Transcription Factor T-bet Resolves Memory B Cell Subsets with Distinct Tissue Distributions and Antibody Specificities in Mice and Humans. Immunity 52, 842-855 e846, doi:10.1016/j.immuni.2020.03.020 (2020).

934 Afgan, E. et al. The Galaxy platform for accessible, reproducible and doi:10.1093/nar/gkw343 (2016). 
93657 Langmead, B. \& Salzberg, S. L. Fast gapped-read alignment with Bowtie 2. Nat

$937 \quad$ Methods 9, 357-359, doi:10.1038/nmeth.1923 (2012).

93858 Langmead, B., Trapnell, C., Pop, M. \& Salzberg, S. L. Ultrafast and memory-

939 efficient alignment of short DNA sequences to the human genome. Genome Biol

940 10, R25, doi:10.1186/gb-2009-10-3-r25 (2009).

94159 Garrison, E., Marth, G. Haplotype-based variant detection from short-read

942 sequencing. arXiv arXiv:1207.3907 (2012).

94360 Wang, K., Li, M. \& Hakonarson, H. ANNOVAR: functional annotation of genetic

$944 \quad$ variants from high-throughput sequencing data. Nucleic Acids Res 38, e164,

945 doi:10.1093/nar/gkq603 (2010). 
bioRxiv preprint doi: https://doi.org/10.1101/2020.11.25.399022; this version posted November 27, 2020. The copyright holder for this preprint

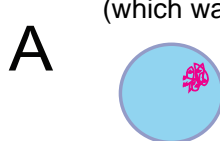

Type I

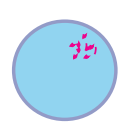

Type II

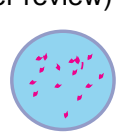

Type III available under aCC-BY-NC-ND 4.0 International license.

B
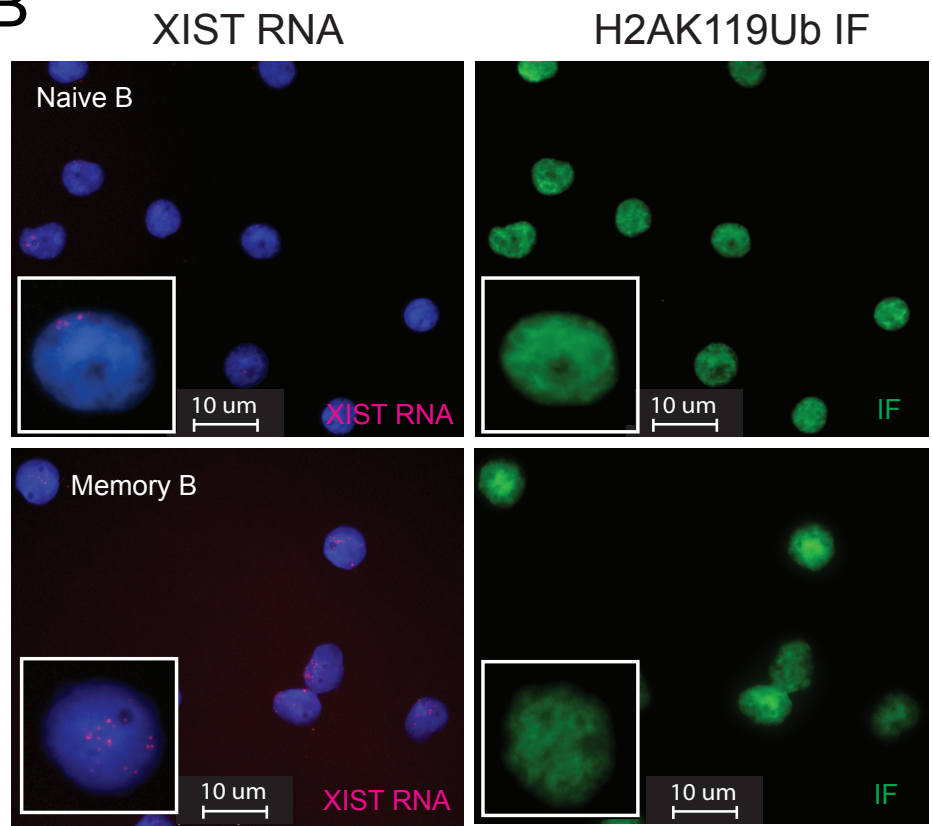

XIST RNA
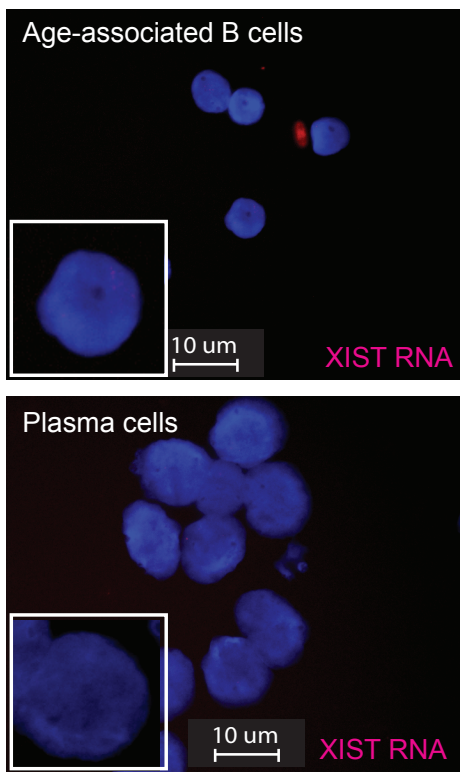

H2AK119Ub IF
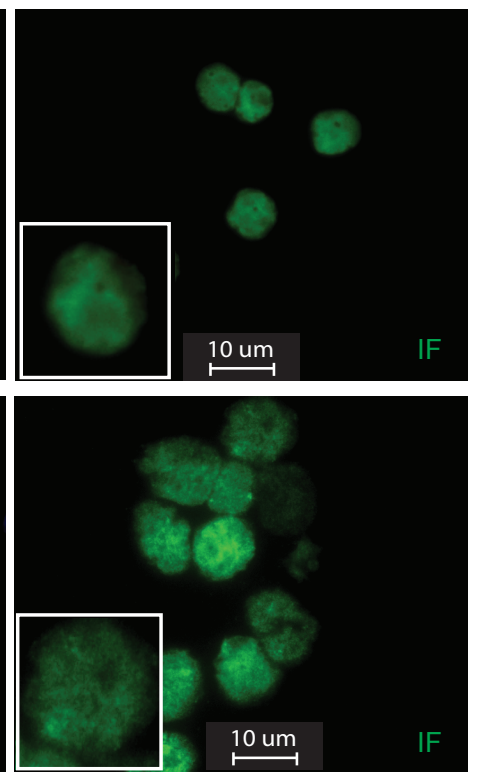

C

XIST RNA localization patterns in circulating B cell subsets

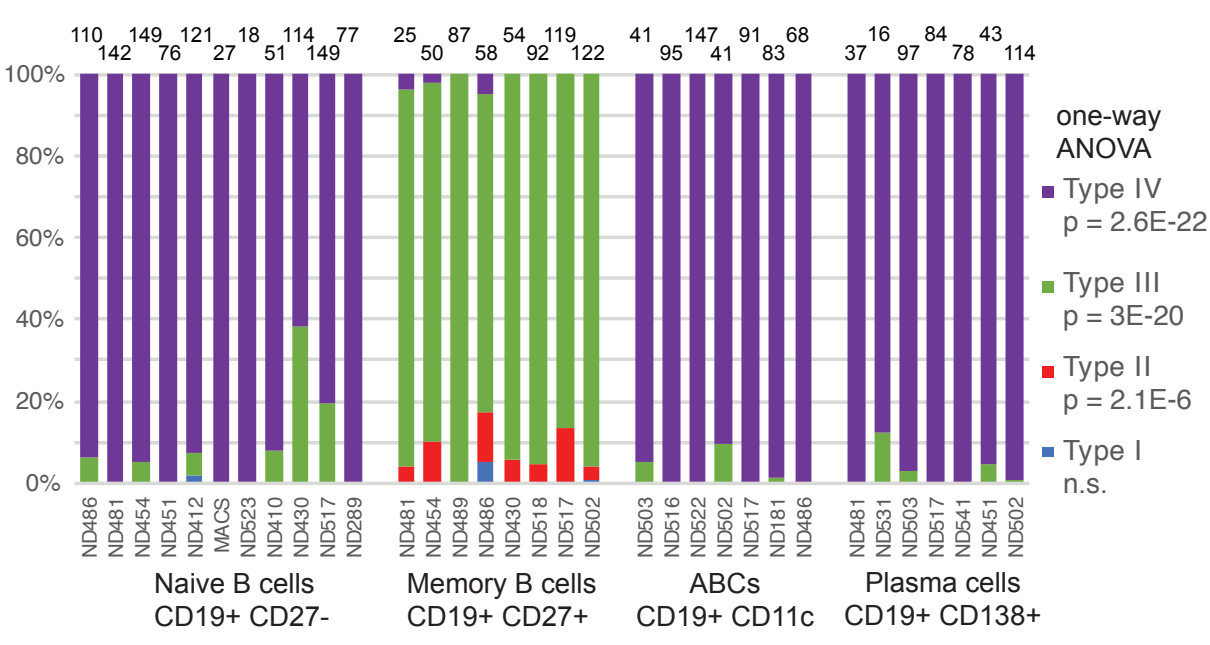

H2AK119Ub foci in circulating B cell subsets

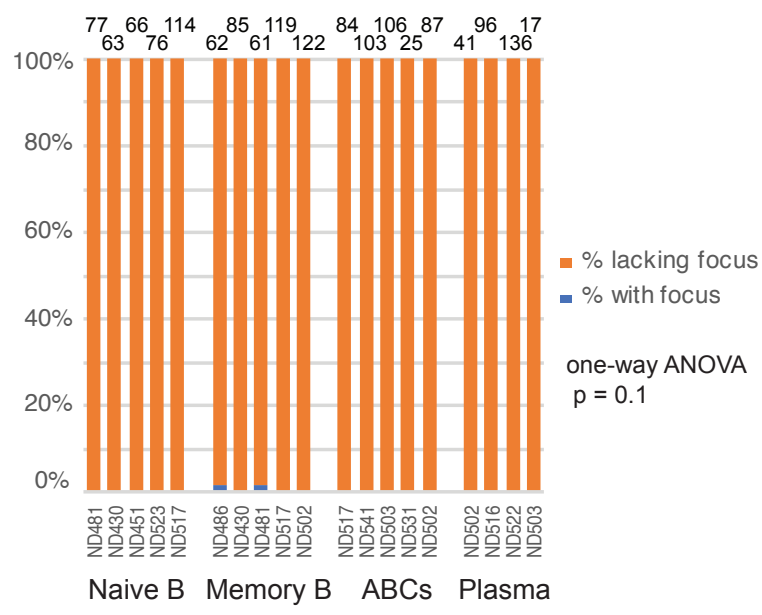

E

XIST RNA Expression in B cell subsets

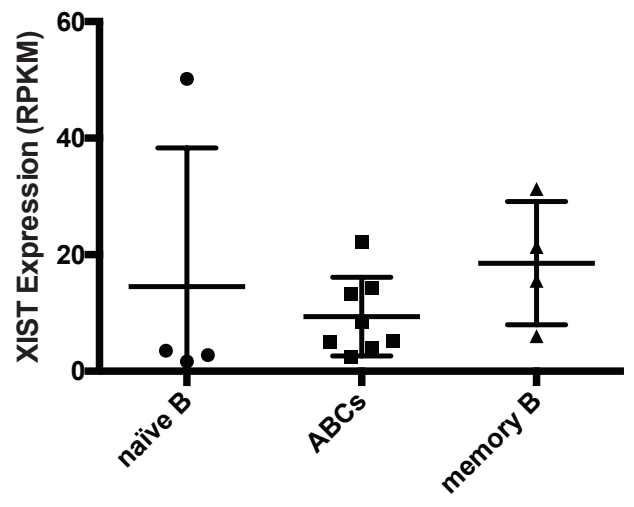


bioRxiv preprint doi: https://doi.org/10.1101/2020.11.25.399022; this version posted November 27, 2020. The copyright holder for this preprint A

(which was not certified by peer review) is the author/funder, who has granted bioRxiv a license to display the preprint in perpetuity. It is made available under aCC-BY-NC-ND 4.0 International license.
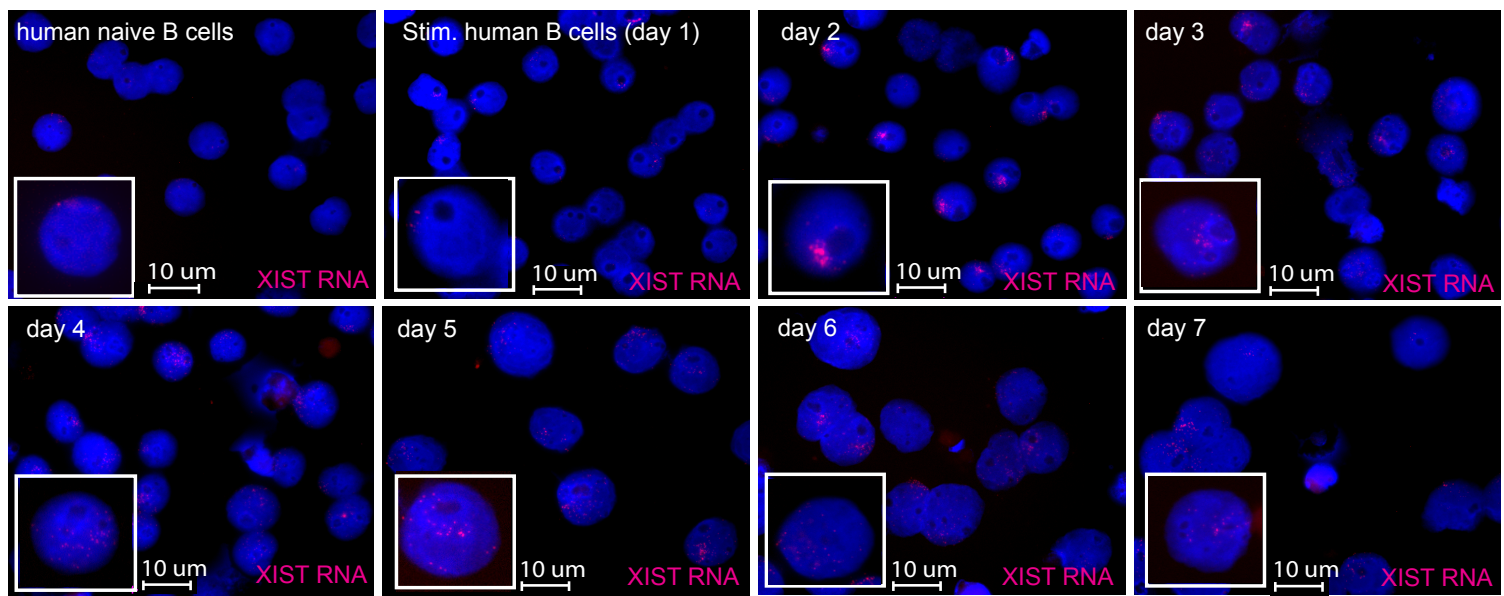

B

C

Time course for XIST RNA localization following in vitro stimulation of B cells
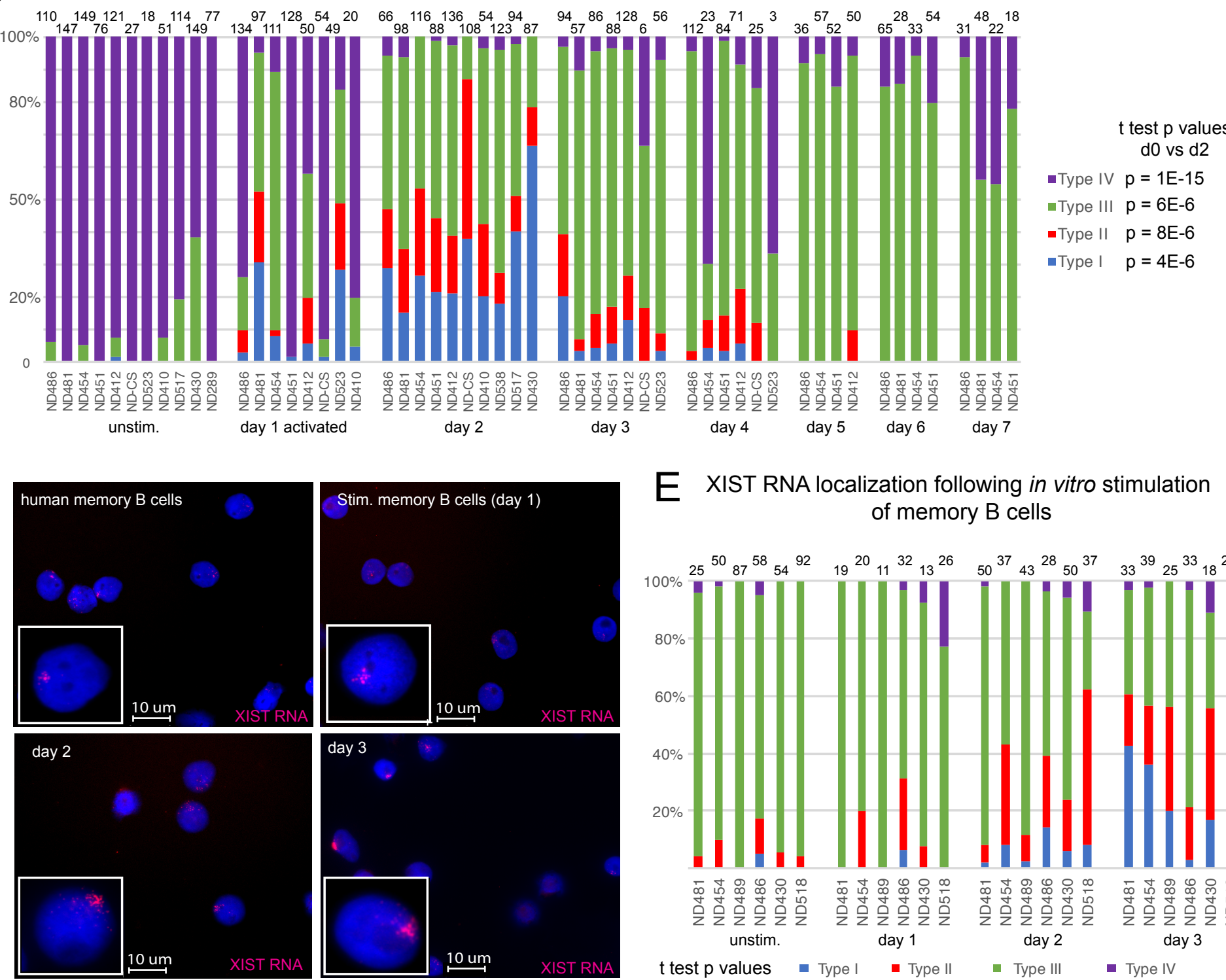

E XIST RNA localization following in vitro stimulation
of memory B cells
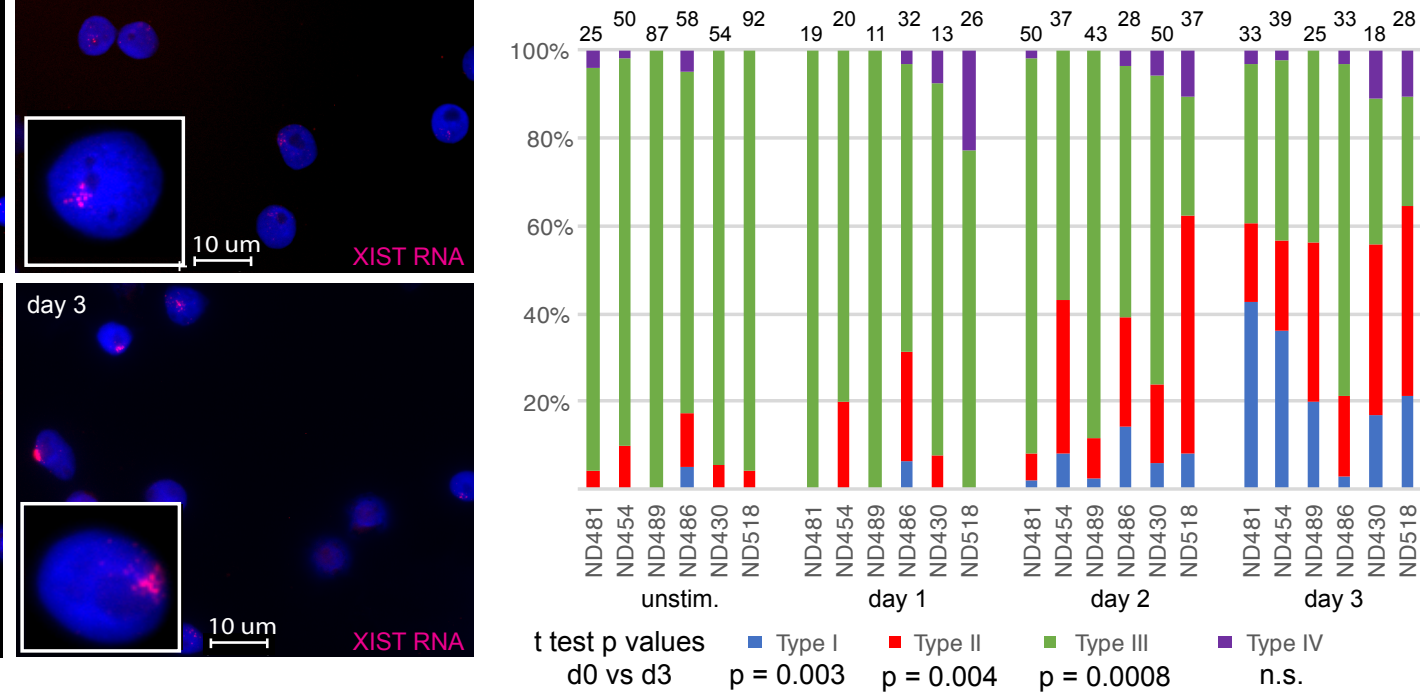

Figure 2 
bioRxiv preprint doi: https://doi.org/10.1101/2020.11.25.399022; this version posted November 27, 2020. The copyright holder for this preprint (which was not certified by peer review) is the author/funder, who has granted bioRxiv a license to display the preprint in perpetuity. It is made available under aCC-BY-NC-ND 4.0 International license.

A

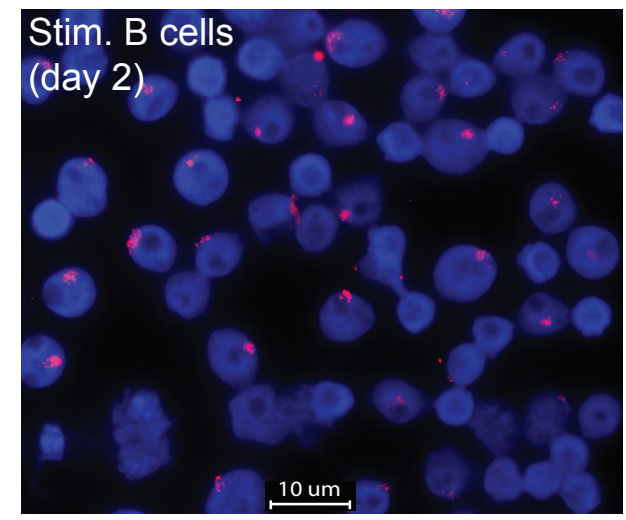

IF

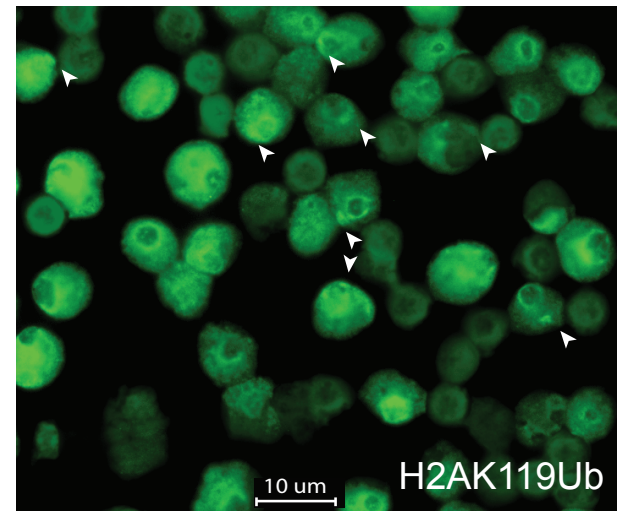

H2AK119Ub foci co-localization with XIST RNA in stimulated $B$ cells

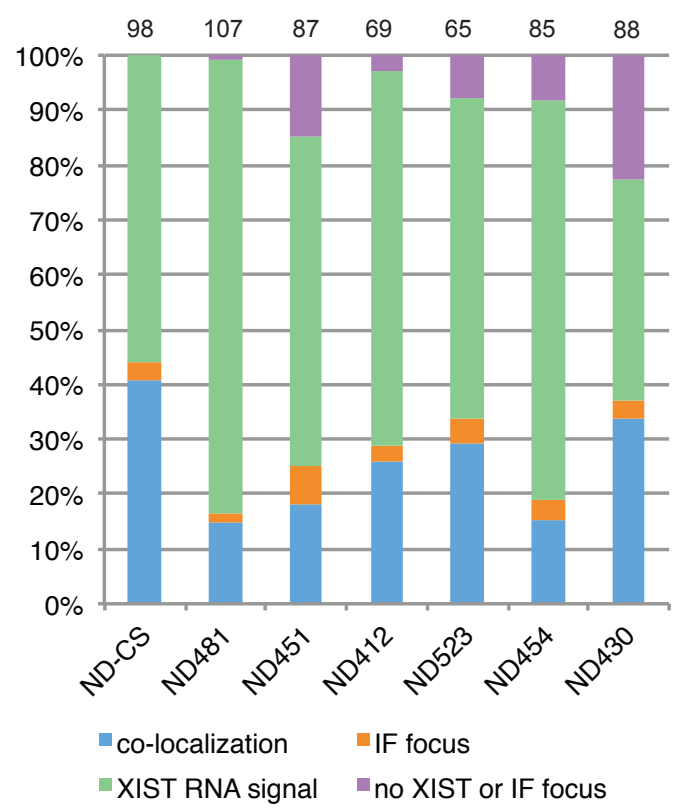

H3K27me3 foci co-localization with XIST

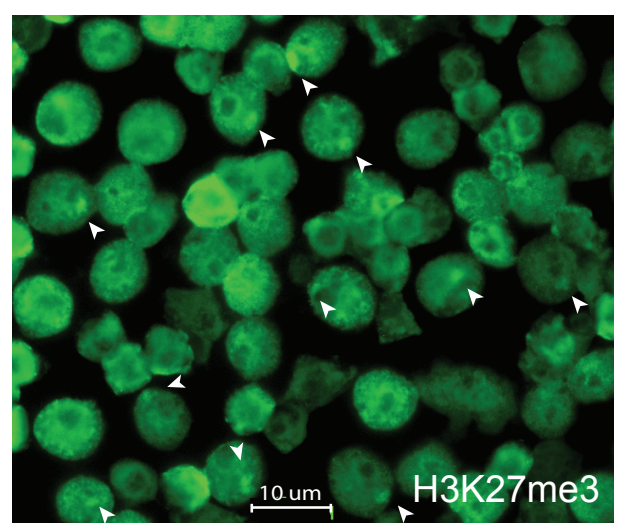

B

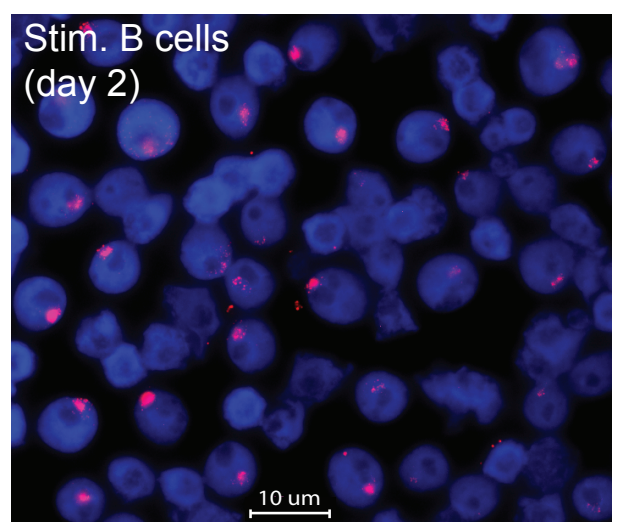
RNA in stimulated $B$ cells

IF

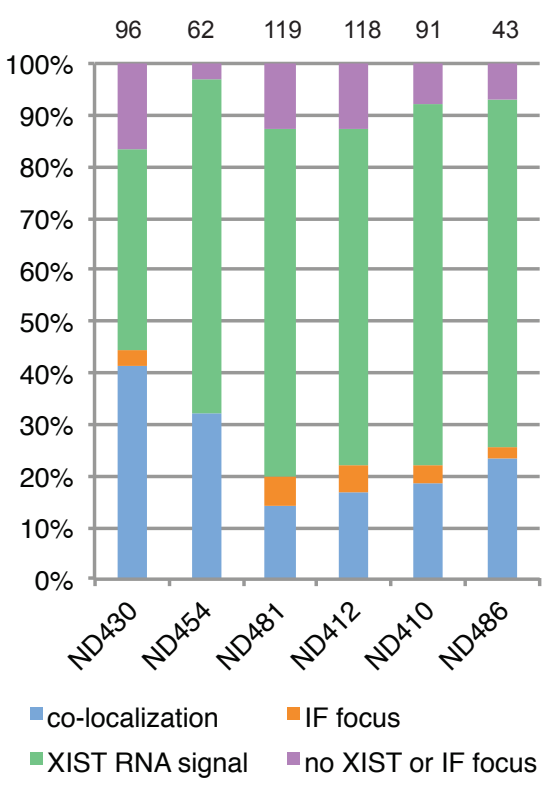

Figure 3 
bioRxiv preprint doi: https://doi.org/10.1101/2020.11.25.399022; this version posted November 27, 2020. The copyright holder for this preprint (which was not certified by peer review) is the author/funder, who has granted bioRxiv a license to display the preprint in perpetuity. It is made available under aCC-BY-NC-ND 4.0 International Alif'X-Finked genes $(\mathbf{n}=391)$

A 7 healthy female donor

30 Memory B cells (CD19+, IgD-, CD27+, IgG+)

30 Naive B cells (CD19+, IgD+, CD27-, CD10-)

30 Plasmablasts (CD19+, IgD-, CD27hi, CD38hi)

27 Transitional B cells (CD19+, IgD+, CD27-, CD10+)

Map reads to reference genome (hg19)

$\downarrow$

SNP detection threshold: at least 10 reads for 1 SNP

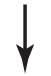

Determine allelic expression of each SNP (mono vs biallelic)

monoallelic: if $\geq 90 \%$ of the reads have the same SNP

$\downarrow$

$\mathrm{XCl}$ escape genes identified as biallelic expression of $>50 \%$ of SNPs in the gene

Detected 6,816 X-linked SNPs 391 unique $X$-linked genes Average of 17 SNPs/gene
36 X-linked genes escape $\mathrm{XCl}$ in at least 1 human $\mathrm{B}$ cell subset (biallelic in 2+ samples)

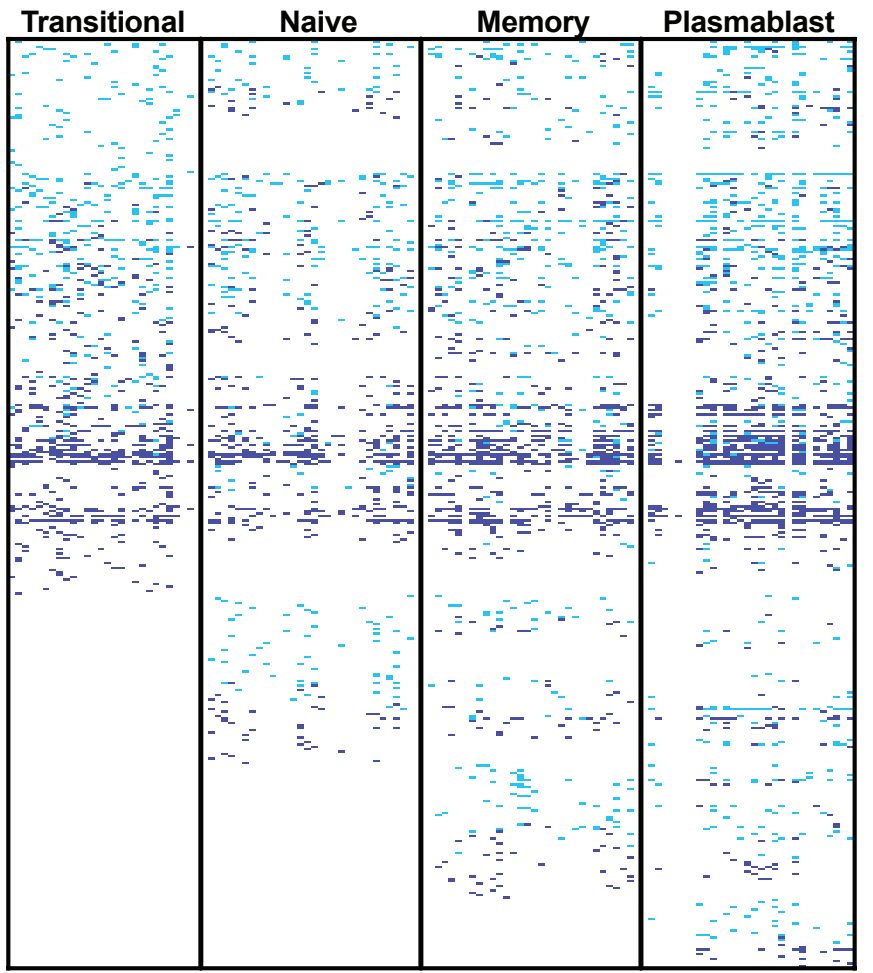

D

Biallelic

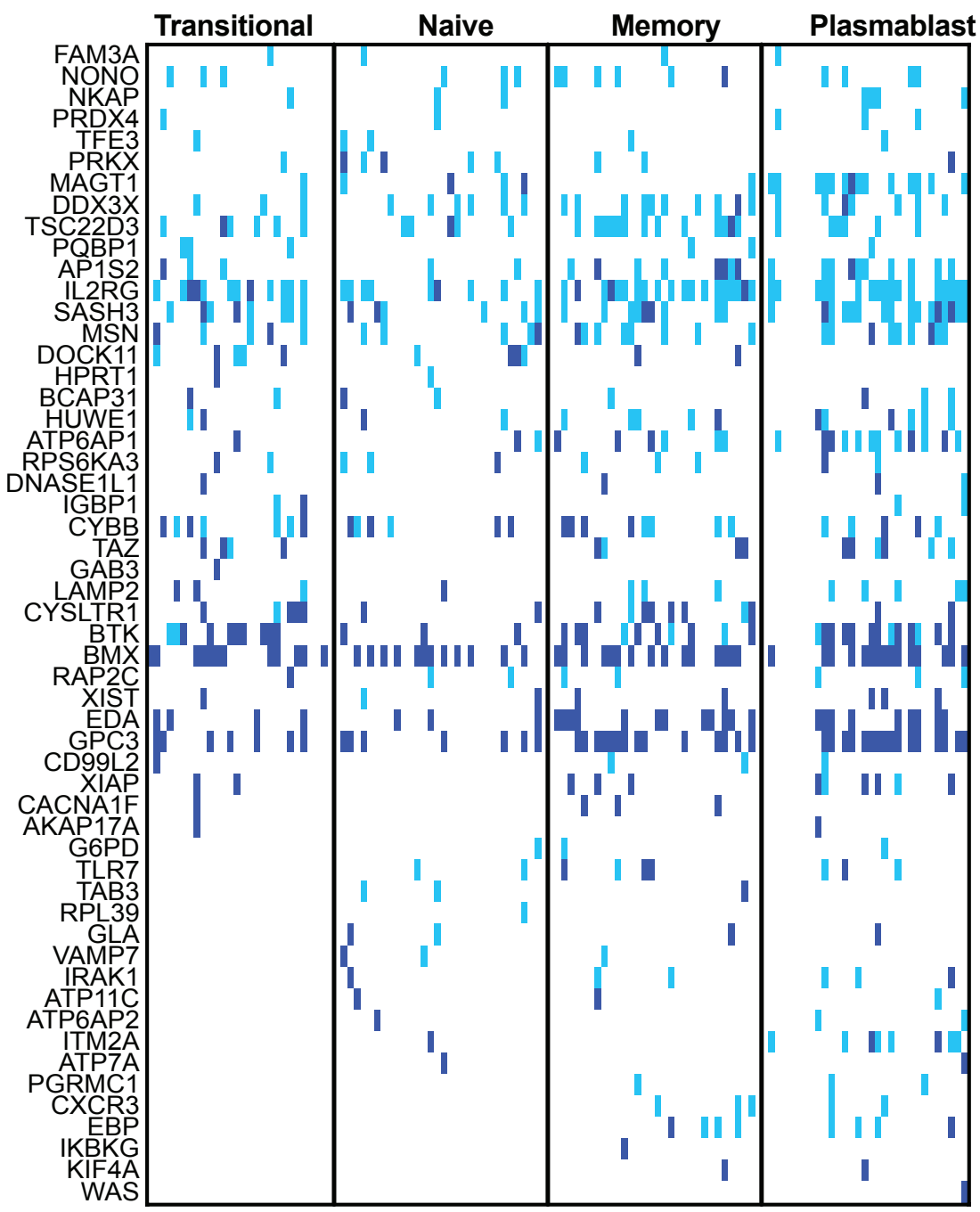

All $X$-linked immunity-related genes that are biallelic or monoallelic in at least $1 \mathrm{~B}$ cell subset

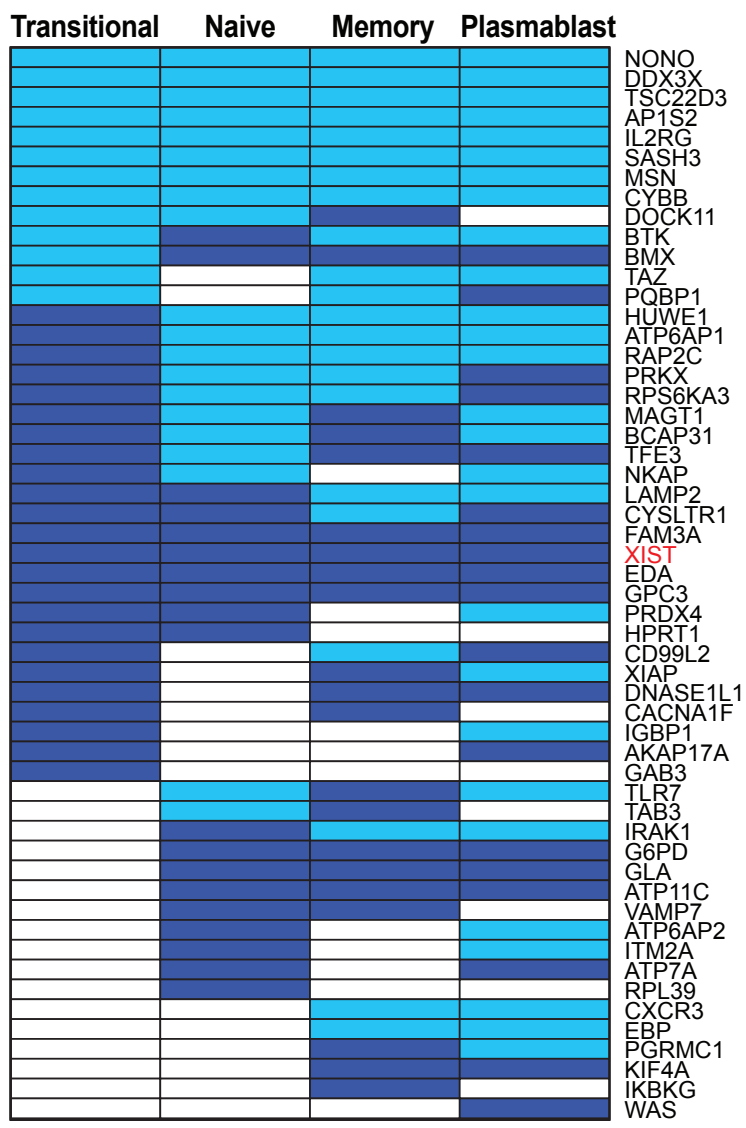

Figure 4 
bioRxiv preprint doi: https://doi.org/10.1101/2020.11.25.399022; this version posted November 27, 2020. The copyright holder for this preprint (which was not certified by peer review) is the author/funder, who has granted bioRxiv a license to display the preprint in perpetuity. It is made A available under aCC-BY-NC-ND 4.0 International license.
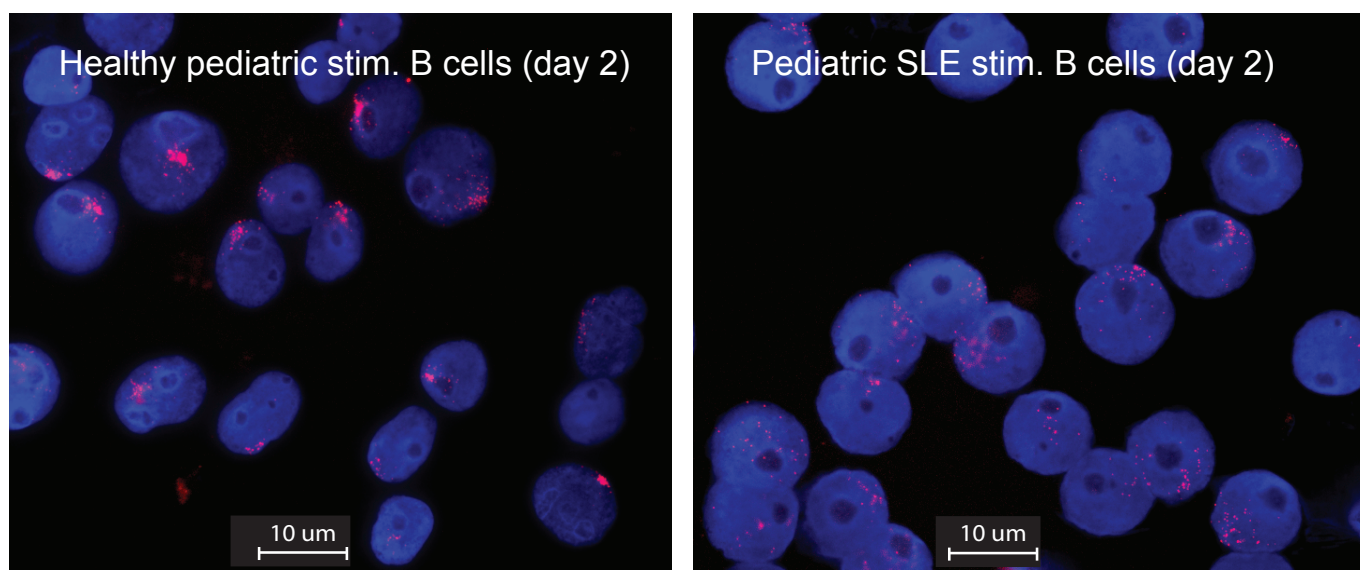

B
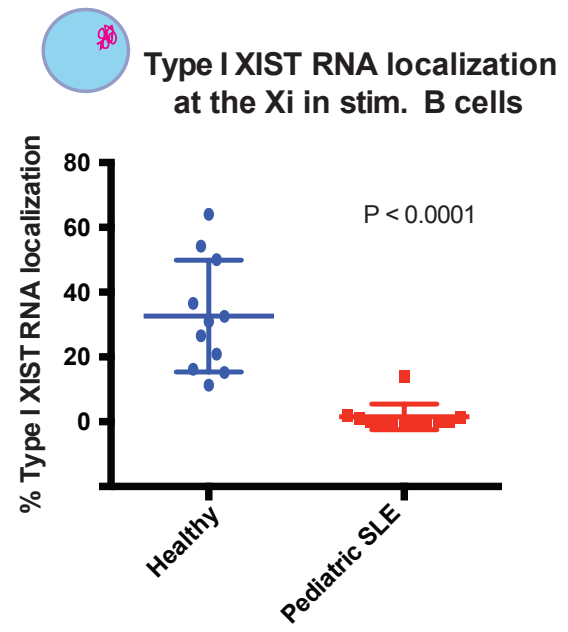

Type II XIST RNA localization at the $\mathrm{Xi}$ in stim. $\mathrm{B}$ cells

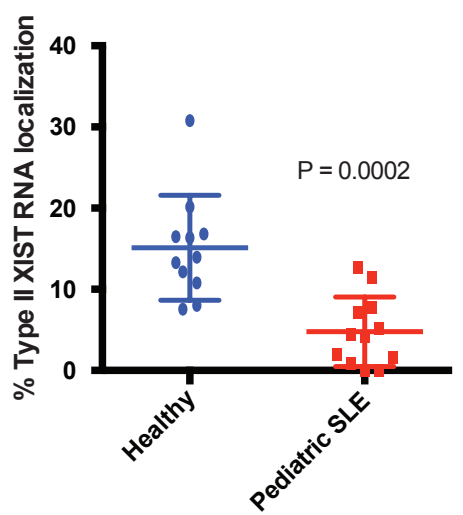

Absence of XIST RNA signal (Type IV) in stim. B cells

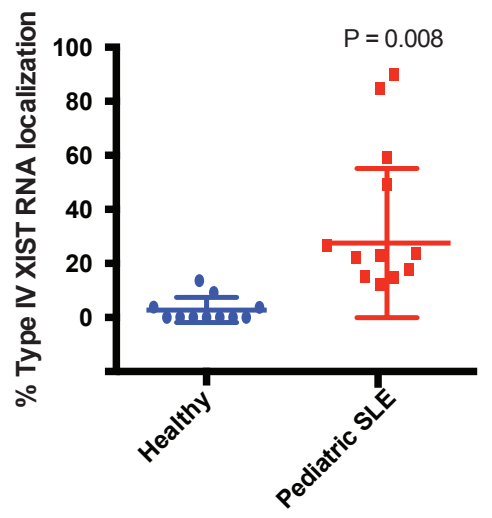

C H2AK119Ub foci in naive and in vitro stim. B cells from pediatric SLE patients

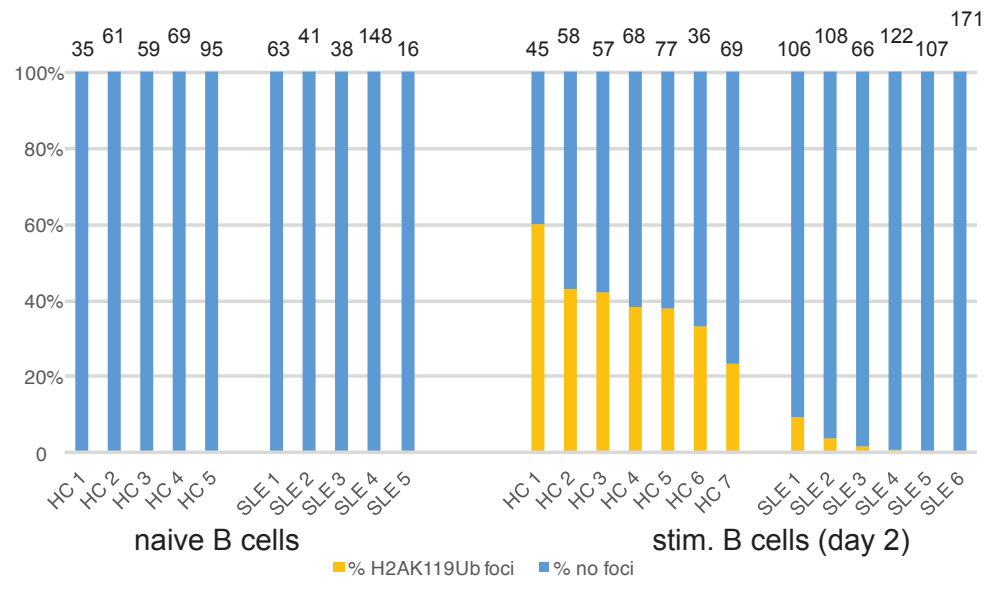

t-test for HC vs SLE: $p=8.9 E-6$ 
bioRxiv preprint doi: https://doi.org/10.1101/2020.11.25.399022; this version posted November 27, 2020. The copyright holder for this preprint (which was not certified by peer review) is the author/funder, who has granted bioRxiv a license to display the preprint in perpetuity. It is made available under aCC-BY-NC-ND 4.0 International license.

A
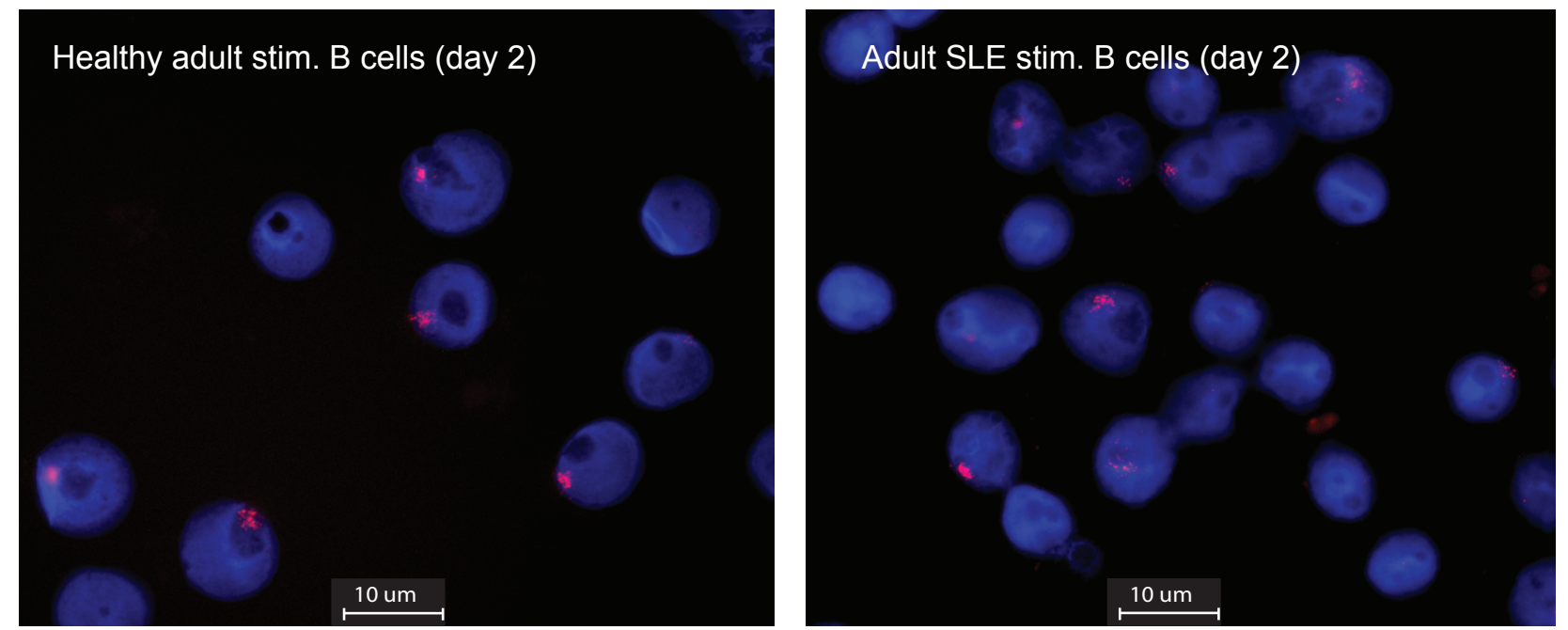

B
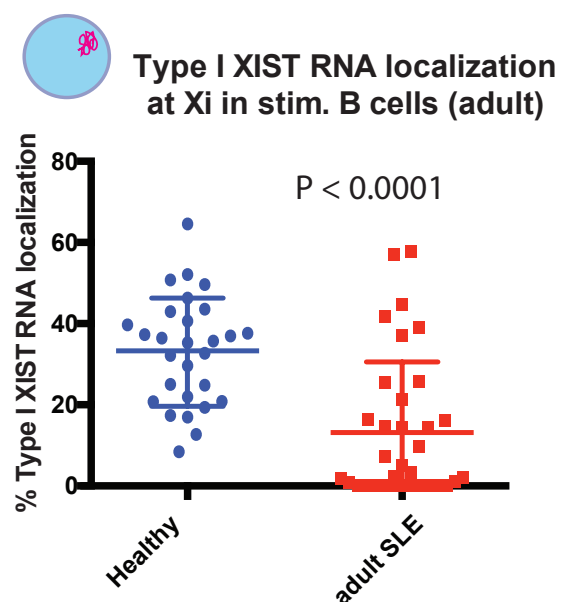

Type II XIST RNA localization at $\mathrm{X}$ in stim. $B$ cells (adult)

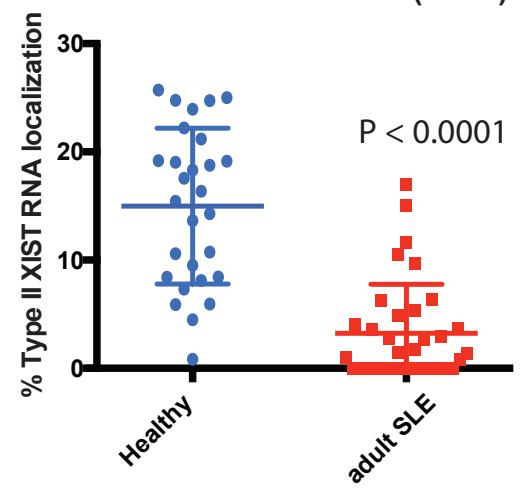

Type IV XIST RNA localization at $\mathrm{X}$ in stim. B cells (adult)

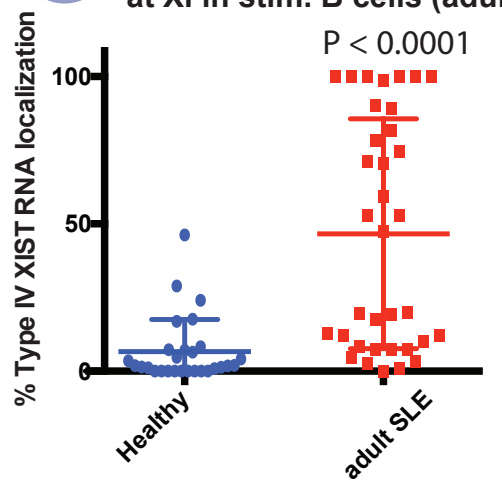

Co-localization of XIST RNA and H2AK119Ub foci in adult SLE patients (in vitro stim. B cells)
$51 \quad 45$
$118 \quad 85$
$97 \quad 53$
$113 \quad 83 \quad 63$
58
$61 \quad 98$
$8 \quad 170 \quad 104 \quad 217 \quad 140 \quad 264 \quad 128$

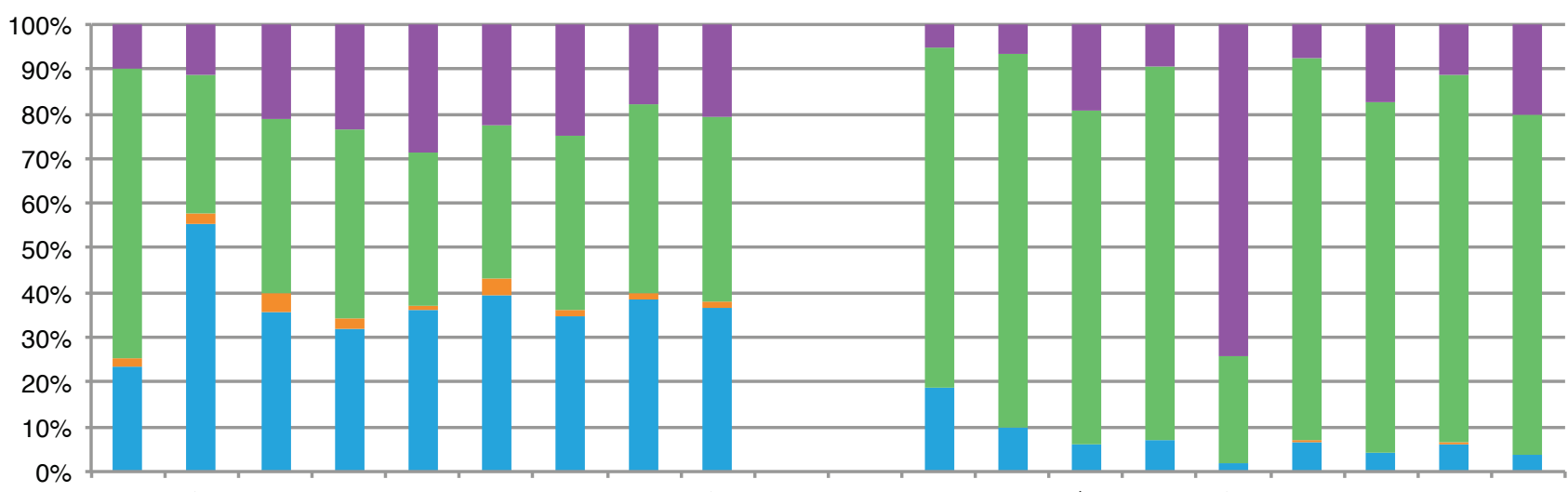

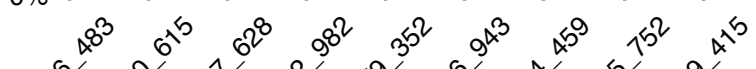

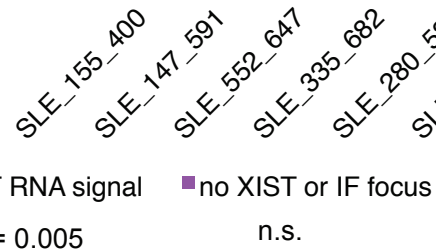


bioRxiv preprint doi: https://doi.org/10.1101/2020.11.25.399022; this version posted November 27, 2020. The copyright holder for this preprint (which was not certified by peer review) is the author/funder, who has granted bioRxiv a license to display the preprint in perpetuity. It is made

A X-linked DEGs $(n=53)$ for Activated $B$ cells

SLE (F) Healthy (F)

\section{6}

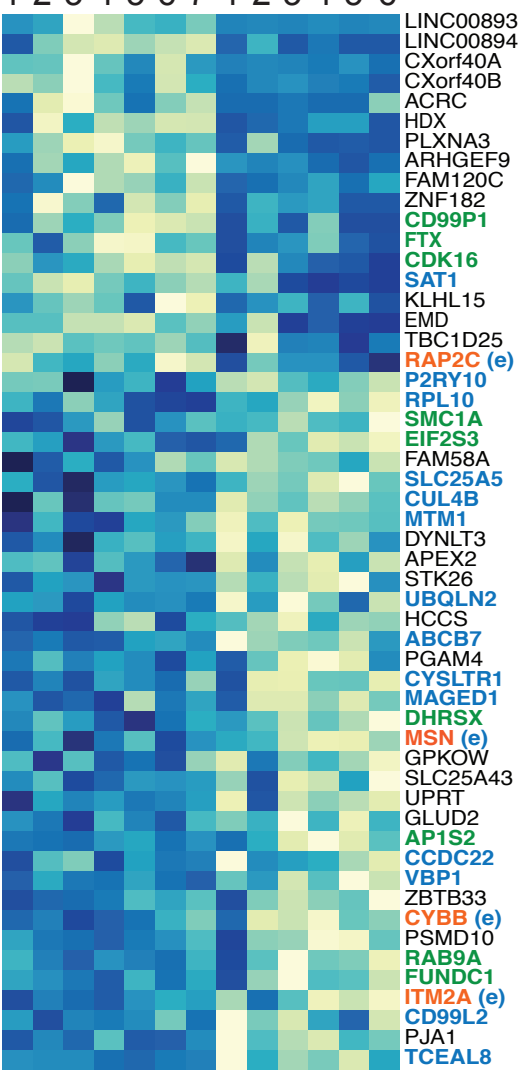

$-2.0$

0

2.0

B

X-linked genes in SLE Activated B cells

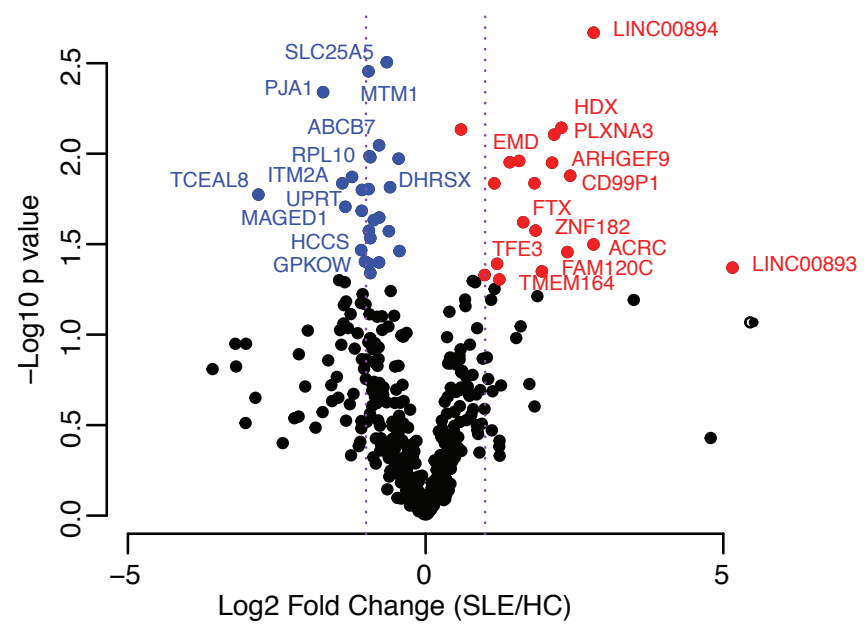

C $\mathrm{BY}$-NCNDT 4 R International license.

$(\mathrm{n}=80)$ in Activated B cells

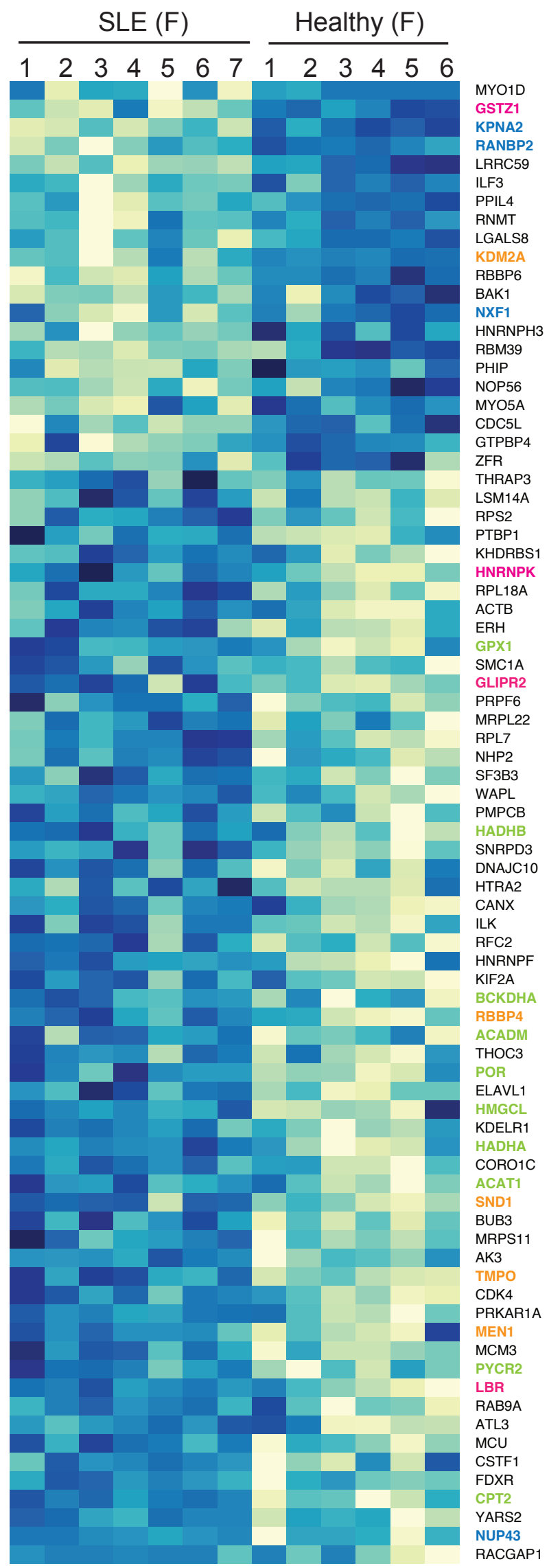

